\title{
Compact covering mappings and cofinal families of compact subsets of a Borel set
}

by

\author{
G. Debs and J. Saint Raymond (Paris)
}

\begin{abstract}
Among other results we prove that the topological statement "Any compact covering mapping between two $\Pi_{3}^{0}$ spaces is inductively perfect" is equivalent to the set-theoretical statement " $\forall \alpha \in \omega^{\omega}, \omega_{1}^{L(\alpha)}<\omega_{1}$ "; and that the statement "Any compact covering mapping between two coanalytic spaces is inductively perfect" is equivalent to "Analytic Determinacy". We also prove that these statements are connected to some regularity properties of coanalytic cofinal sets in $\mathcal{K}(X)$, the hyperspace of all compact subsets of a Borel set $X$.
\end{abstract}

This work is a continuation of [2] and [3] which were motivated by the following topological problem:

Problem 1. Is any compact covering mapping $f: X \rightarrow Y$ between two Borel spaces inductively perfect?

We recall some basic definitions:

The mapping $f: X \rightarrow Y$ is said to be compact covering if any compact subset of $Y$ is the image of some compact subset of $X$.

The mapping $f: X \rightarrow Y$ is said to be inductively perfect if there exists in $X$ a (necessarily closed) subset $X^{\prime}$ such that the restriction $f^{\prime}$ of $f$ to $X^{\prime}$ is a perfect mapping onto $Y$ (i.e. the inverse image under $f^{\prime}$ of every compact subset of $Y$ is compact).

The spaces $X$ and $Y$ considered in Problem 1 are supposed to be metrizable and separable; and in this context, it is shown in [2] that one can reduce the general case to the case where the spaces are zero-dimensional, hence subsets of the Cantor space $2^{\omega}$. Also in what follows a topological space will always be viewed as a subset of $2^{\omega}$ endowed with the induced topology. In particular by a "Borel space", "coanalytic space" etc. we simply mean a Borel or coanalytic subset of $2^{\omega}$. For descriptive classes we follow standard

2000 Mathematics Subject Classification: Primary 03E15; Secondary 03E45, 54H05. 
notations from Effective Set Theory (see Section 1.1 for more details). Notice that since the ambient space is always assumed to be $2^{\omega}$, the class $\mathbf{\Pi}_{2}^{0}=\mathbf{G}_{\delta}$ is here the topological class of all zero-dimensional Polish spaces, and the class $\boldsymbol{\Sigma}_{2}^{0}=\mathbf{K}_{\sigma}$ is the topological class of all zero-dimensional $\sigma$-compact spaces.

For a detailed discussion of Problem 1 we refer the reader to [3]. We list here some of the basic results:

(a) Any compact covering mapping from a $\boldsymbol{\Pi}_{2}^{0}$ space onto any space is inductively perfect.

(b) Any compact covering mapping from any space onto a $\boldsymbol{\Sigma}_{2}^{0}$ space is inductively perfect.

(c) If we assume $\operatorname{Det}\left(\boldsymbol{\Sigma}_{1}^{1}\right)\left(\boldsymbol{\Sigma}_{1}^{1}\right.$ determinacy) then any compact covering mapping from a $\boldsymbol{\Pi}_{1}^{1}$ space onto a $\boldsymbol{\Pi}_{1}^{1}$ space is inductively perfect.

(d) In Gödel's universe L, there exists a compact covering mapping $f$ : $X \rightarrow Y$ between two Borel spaces which is not inductively perfect; moreover $X$ can be chosen to be the intersection of $a \boldsymbol{\Pi}_{2}^{0}$ and $a \boldsymbol{\Sigma}_{2}^{0}$ set, and $Y$ can be chosen to be a $\Pi_{2}^{0}$ set.

Property (a) was proved several years ago by Christensen [1] and by the second author [9] independently, whereas (b) is a much more recent result due to Ostrovskiû [8] (also to Just and Wicke [5] in the particular case where the range space is countable); (c) is from [2] and (d) is from [3]. In particular this gives an example of a "natural" topological property of Borel sets which is not decidable in ZFC.

More generally given two classes $\mathcal{X}$ and $\mathcal{Y}$ of spaces one can ask whether any compact covering mapping from a space in $\mathcal{X}$ to a space in $\mathcal{Y}$ is inductively perfect. Thus Problem 1 concerns the case $\mathcal{X}=\mathcal{Y}=\Delta_{1}^{1}$; but this symmetrical hypothesis on $\mathcal{X}$ and $\mathcal{Y}$ is misleading, and as we shall see the domain space and the range space play totally different roles in this problem. Also for "reasonable" classes $\mathcal{Y}$ we have the following general property:

- If any compact covering mapping from a $\boldsymbol{\Delta}_{1}^{1}$ space onto a space in $\mathcal{Y}$ is inductively perfect, then the same holds for any mapping from a $\boldsymbol{\Pi}_{1}^{1}$ space onto a space in $\mathcal{Y}$.

In fact this is proved in [3], Theorem 6.5 , when $\mathcal{Y}=\Pi_{2}^{0}$, which was the natural context of that work. However the argument is based on some stability properties of the class $\boldsymbol{\Pi}_{2}^{0}$, which are also shared by each of the following classes: $\boldsymbol{\Sigma}_{\xi}^{0}, \Pi_{\xi}^{0}, \boldsymbol{\Delta}_{1}^{1}, \boldsymbol{\Pi}_{1}^{1}$.

In particular it follows from this property that the natural setting for our study is to consider mappings $f: X \rightarrow Y$ where $X$ is a $\Pi_{1}^{1}$ space and $Y$ is a $\boldsymbol{\Delta}_{1}^{1}$ space. 
Another fundamental remark from [3] is that Problem 1 is intimately related to the study of the hyperspace $\mathcal{K}(Y)$ of all compact subsets of the range space $Y$, endowed with the Hausdorff topology. To explain this, let us first fix some terminology:

A subset $\mathcal{A}$ of a hyperspace $\mathcal{K}(Z)$ is called cofinal if it is cofinal for the inclusion relation $\subset$, that is, if

$$
\forall S \in \mathcal{K}(Z), \exists T \in \mathcal{A}, \quad S \subset T .
$$

A domination function for the cofinal set $\mathcal{A}$ is a mapping $f: \mathcal{K}(Z) \rightarrow \mathcal{A}$ such that

$$
\forall S \in \mathcal{K}(Z), \quad f(S) \supset S .
$$

By the Axiom of Choice any cofinal set $\mathcal{A}$ admits a domination function; we say that $\mathcal{A}$ is continuously cofinal if it admits a continuous domination function. In [3] we proved the following surprising result:

- Any analytic cofinal subset of $\mathcal{K}(Z)$ for $Z \boldsymbol{\Pi}_{2}^{0}$ is continuously cofinal.

Notice that if a hyperspace $\mathcal{K}(Z)$ contains an analytic cofinal subset $\mathcal{A}$ then since we can write $\mathcal{K}(Z)=\left\{S \in \mathcal{K}\left(2^{\omega}\right): \exists T \in \mathcal{A}, S \subset T\right\}$ it follows that $\mathcal{K}(Z)$ is also analytic, hence by a basic result of Christensen and Saint Raymond ([9] or [1]), $Z$ is necessarily $\Pi_{2}^{0}$; this result is the origin of property (a) above.

When $Z$ is a general Borel or coanalytic space the following problem arises naturally:

Problem 2. Is any coanalytic cofinal subset of $\mathcal{K}(Z)$, for $Z$ Borel, continuously cofinal?

We now explain the connection between these two problems:

Given any compact covering mapping $f: X \rightarrow Y$ one can code (see the proof of [3], Theorem 6.4) the hyperspace $\mathcal{K}(X)$ by a cofinal subset $\mathcal{A}$ of $\mathcal{K}(Y)$ in such a way that:

- If $\mathcal{A}$ is continuously cofinal then $f$ is inductively perfect.

Conversely given any cofinal subset $\mathcal{A}$ of $\mathcal{K}(Z)$ if we consider the set $\mathcal{H}=\{(S, T) \in \mathcal{K}(Z) \times \mathcal{A}: S \subset T\}$ then the mapping $f: \mathcal{H} \rightarrow \mathcal{K}(Z)$ obtained by restricting to $\mathcal{H}$ the canonical projection on the first factor $(f(S, T)=S)$ is compact covering; moreover one can easily prove that:

- If $f$ is inductively perfect then $f$ admits a domination function of the first Baire class,

but one cannot ensure by general arguments the existence of a continuous domination function.

However for mappings with values in a $\boldsymbol{\Pi}_{2}^{0}$ space we proved in [3]: 
ThEOREM A. The following are equivalent:

(i) For all $\alpha \in \omega^{\omega}$ the set $\omega^{\omega} \cap L(\alpha)$ is $\leq^{\star}$-bounded in $\omega^{\omega}$.

(ii) Any compact covering mapping from a $\boldsymbol{\Pi}_{1}^{1}$ space onto a $\boldsymbol{\Pi}_{2}^{0}$ space is inductively perfect.

(iii) Any $\boldsymbol{\Pi}_{1}^{1}$ cofinal subset of $\mathcal{K}(Z)$ in a $\boldsymbol{\Pi}_{2}^{0}$ space $Z$ is continuously cofinal.

(iv) Any $\boldsymbol{\Pi}_{1}^{1}$ cofinal subset of $\mathcal{K}\left(\omega^{\omega}\right)$ admits a Borel domination function. Here $\leq^{\star}$ denotes the relation of eventual domination of sequences in $\omega^{\omega}$.

In this paper we prove similar equivalences for mappings with values in a $\boldsymbol{\Pi}_{3}^{0}$ space, or in a $\boldsymbol{\Pi}_{1}^{1}$ space:

TheORem B. The following are equivalent:

(i) For all $\alpha \in \omega^{\omega}$ the set $\omega^{\omega} \cap L(\alpha)$ is countable.

(ii) Any compact covering mapping from a $\boldsymbol{\Pi}_{1}^{1}$ space onto a $\boldsymbol{\Pi}_{3}^{0}$ space is inductively perfect.

(iii) Any compact covering mapping from a $\mathbf{\Pi}_{3}^{0}$ space onto a $\boldsymbol{\Pi}_{3}^{0}$ space is inductively perfect.

Theorem C. The following are equivalent:

(i) $\operatorname{Det}\left(\boldsymbol{\Sigma}_{1}^{1}\right)$.

(ii) Any compact covering mapping from a $\boldsymbol{\Pi}_{1}^{1}$ space onto a $\boldsymbol{\Pi}_{1}^{1}$ space is inductively perfect.

(iii) Any $\boldsymbol{\Pi}_{1}^{1}$ cofinal subset of $\mathcal{K}(Z)$ in any $\Pi_{1}^{1}$ space $Z$ is continuously cofinal.

(iv) Any $\Pi_{1}^{1}$ cofinal subset of $\mathcal{K}(\mathbb{Q})$ admits a Borel domination function, where $\mathbb{Q}$ denotes a copy of the set of all rational numbers.

In particular, Problems 1 and 2 get affirmative answers under analytic determinacy.

As one sees, the analogy between Theorems $\mathrm{C}$ and $\mathrm{A}$ is more complete than between Theorems B and A. The main reason behind this fact is a closure property of the classes $\boldsymbol{\Pi}_{2}^{0}$ and $\boldsymbol{\Pi}_{1}^{1}$ : If a space $X$ is in one of these classes then the hyperspace $\mathcal{K}(X)$ is also in the same class. This is not satisfied by the class $\boldsymbol{\Pi}_{3}^{0}$, and for this specific reason the proof of Theorem $\mathrm{B}$ is much more delicate than that of Theorem C.

These results are proved in Section 7. For both theorems the proof decomposes in two main parts, which we now briefly comment on:

(I) Proving that (i) is sufficient to ensure the other conditions.

(II) Proving that (i) is necessary to ensure the other conditions.

In the case of Theorem C, part (I) follows essentially from previous results of [2] and [3]. In the case of Theorem B we proceed similarly to the proof 
of Theorem A in [2], by proving first some "continuous lifting property" over $\Pi_{3}^{0}$ sets; and for this we introduce a Borel game adapted to the new situation. However the arguments make use of totally new ideas. In fact in both situations (Theorems A and B) one is reduced to constructing, from some strategy $\sigma$ in the game, a "large" compact set $K$ included in a given Borel set $Y$. Controlling such a construction when $Y$ is $\boldsymbol{\Pi}_{2}^{0}$ is quite standard, but totally nontrivial when $Y$ is $\boldsymbol{\Pi}_{3}^{0}$. In general in such situations one parametrizes the Borel set $Y$ as the projection of some $\boldsymbol{\Pi}_{2}^{0}$ set $Y^{\prime}$, and constructs the compact set $K$ as the projection of a compact subset $K^{\prime}$ of $Y^{\prime}$. But, for deep reasons, in the present situation this procedure is strictly impossible to realize, and one has to ensure "by hand" the condition $K \subset Y$ when $Y$ is $\boldsymbol{\Pi}_{3}^{0}$. The construction relies on a very technical analysis of the strategy $\sigma$. Also to explain partly this analysis, we shall first treat in Section 4 the simpler case when $Y$ is $\boldsymbol{\Sigma}_{2}^{0}$ and for which the corresponding "lifting property" can be proved in ZFC, without any extra assumption. The case when $Y$ is $\Pi_{3}^{0}$ is developed in Section 5 , which constitutes the heart of this work. This section makes substantial use of Effective Descriptive Set Theory.

Part (II) is nontrivial in Theorem B and in Theorem C, but the proof in both cases follows the same scheme, and makes use of the fact that in each case condition (i) is equivalent to some regularity property for $\boldsymbol{\Pi}_{1}^{1}$ sets (see Theorems 1.2 and 1.3 for more details). The second ingredient of this part is a construction which assigns to any $\Pi_{1}^{1}$ non-Borel set $A \subset 2^{\omega}$ a cofinal subset $\mathcal{A}$ of $\mathcal{K}(\mathbb{Q})$ which is a kind of replica of $A$. Then in each case one has to derive from a continuous domination function for $\mathcal{A}$ the corresponding regularity property for the $\Pi_{1}^{1}$ set $A$. The construction of the replica $\mathcal{A} \subset \mathcal{K}(\mathbb{Q})$ of $A \subset 2^{\omega}$ is independent of the rest of the paper and is presented in Section 6.

As for Theorem A in [3], one can prove effective (lightface) versions of all these results. In Section 1 we present briefly the main nonelementary descriptive results that we use in this work. However we assume the reader to be familiar with Classical and Effective Descriptive Set Theory as well as basic results on the universe $L$, for example as presented in [7].

\section{Descriptive set theory}

1.1. Descriptive classes. We follow standard notations from Effective Descriptive Set Theory: $\boldsymbol{\Sigma}_{\xi}^{0}$ and $\boldsymbol{\Pi}_{\xi}^{0}$ for the additive and multiplicative Borel classes; $\boldsymbol{\Delta}_{1}^{1}$ for the class of all Borel sets; $\boldsymbol{\Sigma}_{1}^{1}$ for the class of all analytic sets; $\boldsymbol{\Pi}_{1}^{1}$ for the class of all coanalytic sets; $\boldsymbol{\Sigma}_{2}^{1}$ for the class of all projections of coanalytic sets; and $\Sigma_{\xi}^{0}, \Pi_{\xi}^{0}, \Delta_{1}^{1}, \Sigma_{1}^{1}, \Pi_{1}^{1}, \Sigma_{2}^{1}$ for the effective versions of the previous classes. 
We shall also consider the following two classes:

$$
\begin{aligned}
& \mathbf{P}_{\sigma}=\left\{A=B \cap C: B \in \mathbf{\Pi}_{2}^{0} \text { and } C \in \boldsymbol{\Sigma}_{2}^{0}\right\}, \\
& \check{\mathbf{P}}_{\sigma}=\left\{A=B \cup C: B \in \mathbf{\Pi}_{2}^{0} \text { and } C \in \boldsymbol{\Sigma}_{2}^{0}\right\},
\end{aligned}
$$

which will play a major role. Obviously $\check{\mathbf{P}}_{\sigma}$ is the class of all complements of sets in $\mathbf{P}_{\sigma}$, and both classes lie between the Borel classes $\boldsymbol{\Pi}_{2}^{0} \subset \boldsymbol{\Pi}_{3}^{0}$. The class $\mathbf{P}_{\sigma}$ is also sometimes denoted by $D_{2}\left(\boldsymbol{\Sigma}_{2}^{0}\right)$. The notation $\mathbf{P}_{\sigma}$ was introduced in [2] where spaces in $\mathbf{P}_{\sigma}$ are called $\sigma$-Polish. A typical set in $\mathbf{P}_{\sigma}$ and not in $\check{\mathbf{P}}_{\sigma}$ is the product space $\mathbb{Q} \times \omega^{\omega}$ as a subset of $2^{\omega} \times 2^{\omega}$ (since both $\mathbb{Q}$ and $\omega^{\omega}$ embed in $2^{\omega}$ ). These classes appear naturally as "limit classes" in several properties of the hyperspace $\mathcal{K}(X)$ for $X$ Borel. For example the following two results, which we shall not use in this work, can be found in [10] and [6]:

- The Borel Effros structure of $\mathcal{K}(X)$ is standard iff $X$ is in $\check{\mathbf{P}}_{\sigma}$.

- The $\sigma$-ideal $\mathcal{K}(X)$ is generated by a Borel subset iff $X$ is in $\mathbf{P}_{\sigma}$.

We shall see in Section 7 that the classes $\mathbf{P}_{\sigma}$ and $\check{\mathbf{P}}_{\sigma}$ are also "limit classes" for two instances of Problem 1 above.

We shall use the following basic and classical result of Solovay [13]:

1.2. TheOrem (Solovay). For any $\alpha \in \omega^{\omega}$ the following are equivalent:

(i) $\omega^{\omega} \cap L(\alpha)$ is countable.

(ii) Any uncountable $\Pi_{1}^{1}(\alpha)$ set contains a perfect set.

We shall commonly write condition (i) in the equivalent form " $\omega_{1}^{L(\alpha)}<$ $\omega_{1}$ ". Notice that these statements are equiconsistent with ZFC, unlike those in the following theorem.

The other basic result we need is a combination of two deep theorems due to Martin and Harrington. Before we state them we recall some standard terminology. A reduction of a set $A \subset 2^{\omega}$ to a set $B \subset 2^{\omega}$ is a function $f: 2^{\omega} \rightarrow 2^{\omega}$ such that $A=f^{-1}(B)$; we also say that $A$ is reducible to $B$ by $f$. If $\mathcal{F}$ is a family of functions from $2^{\omega}$ to $2^{\omega}$ and $\Gamma$ is a class of subsets of $2^{\omega}$, let us say that a set $A_{0}$ in $\Gamma$ is $\Gamma$-complete under $\mathcal{F}$-reductions if any $A$ in $\Gamma$ is reducible to $A_{0}$ by a function $f \in \mathcal{F}$; when $\mathcal{F}$ is the set of all continuous functions, we simply say that $A_{0}$ is $\Gamma$-complete.

1.3. Theorem (Martin-Harrington). For any $\alpha \in \omega^{\omega}$ the following are equivalent:

(i) $\operatorname{Det}\left(\Sigma_{1}^{1}(\alpha)\right)$.

(ii) Any $\Pi_{1}^{1}(\alpha)$ non-Borel set is $\Pi_{1}^{1}(\alpha)$-complete (under continuous reductions).

(iii) Any $\Pi_{1}^{1}(\alpha)$ non-Borel set is $\Pi_{1}^{1}(\alpha)$-complete under Borel reductions. 
The only known proof of this theorem is through the following scheme:

$$
\text { (i) } \Rightarrow \text { (ii) } \Rightarrow \text { (iii) } \Rightarrow \text { (i) }
$$

Aside the middle implication which is trivial, the other two implications are very deep results. The first implication is based on a result of Martin ensuring that analytic determinacy implies the determinacy of games which are differences of analytic sets, hence of Wadge games. The last implication is a theorem of Harrington [4] for which we mention a recent "simple" proof by R. Labib Sami [11] avoiding the use of Steel's forcing as in Harrington's initial proof.

In this work we shall make use of the last implication, that is, Harrington's Theorem. However we shall also use other results and remarks from [2] and [3] which already used Martin's Theorem which is the core of the proof of the first implication.

\section{Sequential spaces}

2.1. General notations. For any set $X$ we denote by $\operatorname{Seq}(X)=\bigcup_{n} X^{n}$ the set of all finite sequences in $X$, and by $\prec$ the extension relation. If $s \in X^{n}$ we denote as usual by

- $|s|$ the length of the sequence $s$,

- $\left.s\right|_{m}$ the restriction of $s$ to $m \leq|s|$,

and if $s$ is nonempty by

- $s^{*}$ the sequence obtained by deleting from $s$ the last element, so that $s^{*}=\left.s\right|_{|s|-1}$.

When working in product spaces we shall very often make obvious identifications such as $(X \times Y) \times Z=X \times Y \times Z$ or $\operatorname{Seq}(X \times Y) \subset \operatorname{Seq}(X) \times \operatorname{Seq}(Y)$. For example if $u=(s, t) \in X^{n} \times Y^{n}$ then we write $\left.u\right|_{m}$ for $\left(\left.s\right|_{m},\left.t\right|_{m}\right)$.

2.2. Lexicographical ordering on $\operatorname{Seq}(X)$. If $(X,<)$ is a wellordered space, denote for each $n \in \omega$ by $<^{n}$ the lexicographical ordering of the product space $X^{n}$. One can extend these orderings to a unique ordering that we shall also denote by $<$ in the following way: for any $s, t \in \operatorname{Seq}(X)$ set

$$
s<t \Leftrightarrow\left[|s|<|t| \text { or }\left(|s|=|t|=n \text { and } s<^{n} t\right)\right] .
$$

It is clear that $\operatorname{Seq}(X)$ is then also wellordered by $<$. In particular one can iterate the previous procedure inductively to endow the sets $\operatorname{Seq}(\operatorname{Seq}(X))$, $\operatorname{Seq}(\operatorname{Seq}(\operatorname{Seq}(X))) \ldots$ with canonical wellorderings that will all be denoted by $<$.

In this paper we consider the wellorder thus obtained on the space $\operatorname{Seq}(\operatorname{Seq}(\kappa))$ where $\kappa$ is some ordinal, and to which we refer as the canonical wellordering of $\operatorname{Seq}(\operatorname{Seq}(\kappa))$. 
2.3. Convergence in $\operatorname{Seq}(X)$. If $\left(s_{n}\right)_{n \in \omega}$ is an infinite sequence in $\operatorname{Seq}(X)$ (that is, $s_{n} \in \operatorname{Seq}(X)$ for each $n$ ), we say that the sequence $\left(s_{n}\right)_{n \in \omega}$ is converging if there exists some $\alpha \in X^{\omega}$ such that:

(1) $\lim _{n}\left|s_{n}\right|=\infty$,

(2) $s_{n}(k)=\alpha(k)$ for all $k \in \omega$ and all $n$ large enough.

It is clear that this $\alpha$ is unique; we say that $\left(s_{n}\right)$ converges to $\alpha$ and write $\alpha=\lim _{n} s_{n}$. Notice that from condition (1) it follows that if $k \in \omega$ is fixed then $s_{n}(k)$ is defined for $n$ large enough.

One can easily interpret this convergence notion as a real topological convergence in some product space. We shall not do this but list simply some elementary properties that we will use later.

2.4. Remarks. (a) If there exists some increasing sequence $\left(k_{n}\right)_{n \in \omega}$ in $\omega$ such that $\left.s_{n}\right|_{k_{n}} \prec \alpha$ for all $n$, then $\left(s_{n}\right)$ converges to $\alpha$. Conversely, if $\left(s_{n}\right)$ converges to $\alpha$ then for any increasing sequence $\left(k_{n}\right)_{n \in \omega}$ in $\omega$, we can extract from $\left(s_{n}\right)$ a subsequence $\left(s_{n}^{\prime}\right)$ (converging to $\alpha$ ) satisfying $\left.s_{n}\right|_{k_{n}} \prec \alpha$ for all $n$.

(b) Suppose that $\alpha=\lim _{n} s_{n}$; if all the $s_{n}$ 's are in some tree $S \subset \operatorname{Seq}(X)$ then $\alpha \in[S]$.

(c) Suppose that $X$ is wellordered by $<$. If the sequence $\left(s_{n}\right)$ is such that for all $m<n$ :

(1) $\left|s_{m}\right|<\left|s_{n}\right|$,

(2) the restriction of $s_{n}$ to $\left|s_{m}\right|$ is smaller than $s_{m}$ (lexicographically in $X^{\left|s_{m}\right|}$,

then for all $k$ the sequence $\left(s_{n}(k)\right)_{n}$ is eventually nonincreasing, hence stationary; and $\left(s_{n}\right)$ is convergent.

Finally we shall make wide use of the following compactness property:

2.5. Lemma. Let $\left(s_{n}\right)$ be an infinite sequence in $\operatorname{Seq}(X)$ such that $\left|s_{n}\right| \rightarrow$ $\infty$, and suppose that for any $k \in \omega$ the set $\left\{s_{n}(k): n\right.$ such that $\left.\left|s_{n}\right|>k\right\}$ is finite. Then one can extract from $\left(s_{n}\right)$ an infinite converging subsequence.

Proof. Starting from the initial sequence $\sigma^{(0)}=\left(s_{n}^{(0)}\right)=\left(s_{n}\right)$ one constructs inductively subsequences $\sigma^{(j)}=\left(s_{n}^{(j)}\right)$ such that for all $j, \sigma^{(j+1)}$ is a subsequence of $\sigma^{(j)}$ and the sequence $\left(s_{n}^{(j)}(j)\right)$ is constant in $n$. Then it is clear that the diagonal subsequence $\left(s_{n}^{\prime}\right)=\left(s_{n}^{(n)}\right)$ is converging.

3. The games of type $\Gamma(T)$. For the proofs of the main results of the next two sections we introduce two different games, each adapted to a particular hypothesis, but both of the same type. Also to avoid repetitions we describe in this section the general form of these games and fix some notations and conventions that we will respect in what follows. 
Set $\Sigma=\operatorname{Seq}\left(\omega_{1}\right)$ and let $T$ be a tree on $\{0,1\} \times \Sigma$. By a game of type $\Gamma(T)$ we mean a game in which at each move:

- Player I chooses some element in $\{0,1\}$,

- Player II chooses an element in $\{0,1\} \times \Sigma$.

We identify an infinite run in such a game with a triple $(y, z, \theta) \in 2^{\omega} \times 2^{\omega} \times$ $\Sigma^{\omega}$; we say that $y$ and $z$ are the reals constructed in the run by Player I and by Player II, and we shall refer to the $\theta(n)$ 's as the ordinal moves of Player II in the run.

Rules. To be legal in the game, the run $(y, z, \theta)$ should satisfy for all $n \geq 0$ the following two rules:

$\left(\mathrm{R}_{1}\right) \quad|\theta(n)| \leq n+1$.

$\left(\mathrm{R}_{2}\right) \quad$ If $|\theta(n)|=m$ then $\left(\left.z\right|_{m}, \theta(n)\right) \in T$.

Notice that both rules concern Player II; in particular any move is legal for Player I. In fact in the games that we shall consider later we shall add more rules but they will again concern only Player II. We do not require any particular form for the win condition, which will be specifed in each particular game. Notice that if $\left(r_{n}\right)$ is a sequence of legal finite runs in the game which is converging (in the sense of 2.3) then by Remark 2.4(b) the $\operatorname{limit}_{n} \lim _{n}$ is an infinite run in which the same rules are satisfied.

Finite runs. We always identify a finite run in the game with a triple

$$
(s, t, \theta) \in \operatorname{Seq}(2) \times \operatorname{Seq}(2) \times \operatorname{Seq}(\Sigma)
$$

where $s$ represents the moves of Player I in the run, and $(t, \theta)$ represents the moves of Player II in the run. We always have $|t|=|\theta|$, and $|s|=|t|+1$ or $|t|$ depending on whether the last move in the run is played by Player I or by Player II.

Positions. By a position for Player II in the game we mean a couple

$$
(t, \theta) \in \operatorname{Seq}(2) \times \operatorname{Seq}(\Sigma)
$$

which can be realized as the moves of Player II in some run:

$$
(s, t, \theta) \in \operatorname{Seq}(2) \times \operatorname{Seq}(2) \times \operatorname{Seq}(\Sigma)
$$

where all rules are satisfied (not only $\left(\mathrm{R}_{1}\right)$ and $\left(\mathrm{R}_{2}\right)$ ).

One can also define similarly a position for Player I; but since we never deal with this notion in what follows, by "position" we always mean "position for Player II". The empty set is a position corresponding to the empty run, or to a run with one move made by Player I.

If $\sigma$ is some fixed strategy for Player I in the game, we say that the position $u=(t, \theta)$ is compatible with $\sigma$ if we can find a run $r=(s, t, \theta)$ compatible with $\sigma$. By convention we also require that in this run Player I makes 
the last move, so that $r$ is uniquely determined by $u$, and the correspondence between $u$ and $r$ is one-to-one. The position $\emptyset$ is always compatible with $\sigma$ and corresponds to the run with one move $\sigma(\emptyset)$.

Notations. For any position $u=(t, \theta)$ we set

$$
\pi(u)=t, \quad \bar{\lambda}(u)=\theta .
$$

If moreover $u$ is compatible with some strategy $\sigma$ and is realized by the run $(s, t, \theta)$ with $|s|=|t|+1$ then we write

$$
\sigma(u)=s .
$$

Notice that $|\sigma(\emptyset)|=1$ and $\sigma(u)$ is never empty.

\section{Continuous liftings over $\Sigma_{2}^{0}$ spaces}

4.1. TheOrem. Let $R$ and $Y \times Z$ be two subsets of $2^{\omega} \times 2^{\omega}$ satisfying:

(a) $Y$ is $\boldsymbol{\Sigma}_{2}^{0}, Z$ is $\boldsymbol{\Sigma}_{2}^{1}, R$ is $\boldsymbol{\Delta}_{1}^{1}$,

(b) for every compact $K \subset Y$, there exists $z \in Z$ such that $K \times\{z\} \subset R$. Then there exists a continuous mapping $f: 2^{\omega} \rightarrow 2^{\omega}$ such that $\left.f\right|_{Y} \subset$ $R \cap(Y \times Z)$ where $\left.f\right|_{Y}$ denotes the graph of the restriction of $f$ to $Y$.

The proof of this theorem is easy if $Z$ is assumed to be Borel, but in the general case the problem is to keep the game Borel.

To prove this result we introduce a game of type $\Gamma(T)$ for some tree $T$ such that:

(1) If Player II has a winning strategy then the conclusion of the theorem holds.

(2) If Player I has a winning strategy then there is a compact set $K$ negating hypothesis (b) of the Theorem.

Ensuring (1) will be straightforward from the win condition of the game, and the main point will be to prove (2). In fact given an arbitrary strategy $\sigma$ for Player I we shall define a compact subset $K$ of $2^{\omega}$ such that if $\sigma$ is winning then $K$ negates hypothesis (b).

4.2. Index of an $\omega$-sequence of trees. Let $\mathbf{S}=\left(S_{k}\right)_{k \in \omega}$ be an $\omega$-sequence of trees on $\{0,1\}$. The index of $\mathbf{S}$ is the mapping $\mathbf{k}: \operatorname{Seq}(2) \rightarrow \omega$ defined by

$$
\mathbf{k}(s)= \begin{cases}\min \left\{k<|s|: s \in S_{k}\right\} & \text { if } s \in \bigcup_{k<|s|} S_{k}, \\ |s| & \text { if not. }\end{cases}
$$

Notice that if $s \prec s^{\prime}$ then $\mathbf{k}(s) \leq \mathbf{k}\left(s^{\prime}\right)$. In particular if $s \neq \emptyset$ then $\mathbf{k}\left(s^{*}\right) \leq$ $\mathbf{k}(s)$; and we say that $s$ is critical if $\mathbf{k}\left(s^{*}\right)<\mathbf{k}(s)$.

It is clear that for any $x \in 2^{\omega}$ we have

$$
x \notin \bigcup_{k \in \omega}\left[S_{k}\right] \Leftrightarrow \lim _{n} \mathbf{k}\left(\left.x\right|_{n}\right)=\infty \Leftrightarrow \limsup _{n} \mathbf{k}\left(\left.x\right|_{n}\right)=\infty .
$$


4.3. The game $G^{\prime}$. Since $Z$ is $\boldsymbol{\Sigma}_{2}^{1}$ we can fix a tree $T$ on $2 \times \omega_{1}$ such $Z$ is the projection of $[T]$ on the first factor (see [7], p. 84). We also fix a sequence $\left(S_{k}\right)_{k \in \omega}$ of trees on 2 such that $Y=\bigcup_{k}\left[S_{k}\right]$ and denote by $\mathbf{k}$ the associated index.

We now consider the game $G^{\prime}$ of type $\Gamma(T)$ in which rule $\left(\mathrm{R}_{2}\right)$ is unchanged whereas $\left(\mathrm{R}_{1}\right)$ is replaced by the following rule:

$\left(\mathrm{R}_{1}^{\prime}\right) \quad$ If $(s, t, \theta)$ is a run with $|s|=|t|=|\theta|=n+1$ then

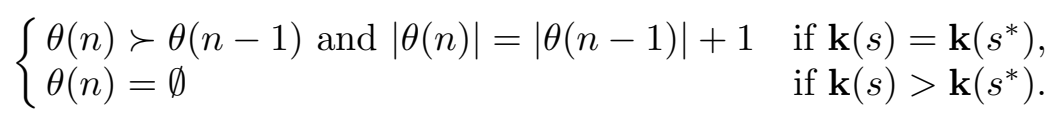

It is easy to check that this rule is stronger than $\left(R_{1}\right)$.

Win condition. Player II wins the infinite run $(y, z, \theta)$ in the game $G^{\prime}$ if

$$
y \notin Y \quad \text { or } \quad(y, z) \in R .
$$

All game-theoretical notions considered in this section are relative to the game $G^{\prime}$.

4.4. Lemma. If Player II has a winning strategy then there exists a continuous mapping $f: 2^{\omega} \rightarrow 2^{\omega}$ such that $\left.f\right|_{Y} \subset R \cap(Y \times Z)$.

Proof. A winning strategy $\tau$ for Player II defines a Lipschitz mapping $y \mapsto(f(y), g(y))$ from $2^{\omega}$ into $2^{\omega} \times \Sigma^{\omega}$ where each $(y, f(y), g(y))=(y, z, \theta)$ is an infinite run compatible with $\tau$, hence won by Player II.

Fix $y \in Y$; it follows from the win condition that $(y, f(y)) \in R$ and all we have to prove is that $f(y) \in Z$. Set

$$
k_{0}=\min \left\{k \in \omega: y \in\left[S_{k}\right]\right\} .
$$

It follows from the definition of the index $\mathbf{k}$ that there exists some $n$ such that $\mathbf{k}\left(\left.y\right|_{m}\right)=k_{0}$ for all $m \geq n$. Let $n_{0}$ be the least such $n$; then by rule $\left(\mathrm{R}_{1}^{\prime}\right)$ there exists a unique $\gamma \in \omega_{1}^{\omega}$ such that $\left.\gamma\right|_{k}=\theta\left(n_{0}+k\right)$ for all $k$, and since by rule $\left(\mathrm{R}_{2}\right)$ we have $\left(\left.z\right|_{k},\left.\gamma\right|_{k}\right) \in T$ for all $k$, it follows that $(z, \gamma) \in[T]$ and $z=f(y) \in Z$.

We now fix a strategy $\sigma$ for Player I in the game. All runs and positions considered from now on will implicitly be assumed to be compatible with $\sigma$.

Our next goal is to define from $\sigma$ a compact set $K$ with the properties announced above. The definition of $K$ will rely on an analysis of the strategy $\sigma$ for which we need to introduce the following notions.

Minimal positions. We say that a position $u$ is minimal if for any position $v$ the following implication holds:

$$
(\sigma(v)=\sigma(u) \text { and } \pi(v)=\pi(u)) \Rightarrow \bar{\lambda}(u) \leq \bar{\lambda}(v)
$$

where as always $\leq$ refers to the canonical lexicographical ordering on $\operatorname{Seq}(\Sigma)$. 
Given any position $v$ it is clear that there exists a unique minimal position $u$ with $\sigma(v)=\sigma(u)$ and $\pi(v)=\pi(u)$; we denote this $u$ by $\mu(v)$.

4.5. Lemma. If $u$ is a minimal position and $v$ is a position extending $u$ then $\mu(v)$ is also an extension of $u$.

Proof. Let $u^{\prime}$ denote the position obtained by restriction of $\mu(v)$ to $|u|$. If $\bar{\lambda}(u)<\bar{\lambda}\left(u^{\prime}\right)$ then we would also have $\bar{\lambda}(v)<\bar{\lambda}(\mu(v))$, which is impossible by the minimality of $\mu(v)$. Hence $\bar{\lambda}(u)=\bar{\lambda}\left(u^{\prime}\right)$ and since $\pi(u)=\pi\left(u^{\prime}\right)$, we have $u=u^{\prime}$ and so $\mu(v) \succ u^{\prime}=u$.

Good positions. We say that a position $u$ is good if for any subposition $v \preceq u$ the following implication holds:

$$
\sigma(v) \text { is critical } \Rightarrow v \text { is minimal }
$$

where "critical" refers to the index $\mathbf{k}$ associated with the fixed sequence $\left(S_{k}\right)_{k \in \omega}$. Set

$$
S=\{\sigma(u): u \text { a good position }\} \cup\{\emptyset\} .
$$

Since any subposition of a good position is clearly good, $S$ is a tree on $\{0,1\}$ and we can define the compact set

$$
K=[S] .
$$

We say that the strategy $\sigma$ takes its values in $Y$ if in any infinite run $(y, z, \theta)$ compatible with $\sigma$ the real $y$ constructed by Player I belongs to $Y$. It follows from the win condition of the game that any winning strategy takes its values in $Y$.

4.6. Lemma. If $\sigma$ takes its values in $Y$ then $K \subset Y$.

Proof. Fix $y \in K=[S]$. For each $n$ the sequence $s_{n}=\left.y\right|_{n+1}$ is in $S$, so we can find a good position $u_{n}=\left(t_{n}, \theta_{n}\right)$ such that $\left|u_{n}\right|=n$ and $s_{n}=\sigma\left(u_{n}\right)$.

Fix $n$ such that $s_{n}$ is critical. We can find $t \in 2^{n}$ such that $\left.t_{m}\right|_{n}=t$ for infinitely many $m$ 's. Now if we fix such an $m$, since $u_{m}$ is a good position and $\sigma\left(\left.u_{m}\right|_{n}\right)=s_{n}$ is critical, the position $\left.u_{m}\right|_{n}$ is minimal and hence there exists $\theta \in \Sigma^{n}$ uniquely determined by $s_{n}$ and $t$ such that $\left.\theta_{m}\right|_{n}=\theta$. Thus if $s_{n}$ is critical then there exists a position $v=(t, \theta)$ such that $\sigma(v)=s_{n}$ and $\left.u_{m}\right|_{n}=v$ for infinitely many $m$ 's.

Suppose now by contradiction that $y \notin Y$. Then the set $N=\{n \in \omega$ : $s_{n}$ is critical $\}$ is infinite, and applying inductively the previous observations one can construct a family $\left(v_{n}\right)_{n \in N}$ of increasing positions $\left(v_{n} \prec v_{n^{\prime}}\right.$ if $\left.n<n^{\prime}\right)$ such that $\sigma\left(v_{n}\right)=s_{n} \prec y$ for all $n \in N$. This clearly defines a unique infinite run compatible with $\sigma$ in which the real constructed by Player I is precisely $y$; and since $y \notin Y$ this contradicts the hypothesis of the lemma. 
4.7. Lemma. If $\sigma$ takes its values in $Y$ then for any $z \in Z$ there exists an infinite run $(y, z, \theta)$ (compatible with $\sigma$ ) such that $y \in K$.

Proof. We say that a position $u$ is critical if $\sigma(u) \in \operatorname{Seq}(2)$ is critical with respect to the index $\mathbf{k}$.

Fix $z$ in $Z$ and let $U=U_{z}$ denote the set of all positions $u$ satisfying $\pi(u) \prec z$. We shall construct a finite sequence $\left(u_{k}\right)_{k \leq n}$ in $U$ satisfying for all $k \leq n$ :

(1) $u_{k-1} \prec u_{k}$

(2) $u_{k}$ is a good position,

(3) $u_{k}$ is critical,

(4) any extension of $u_{n}$ in $U$ is noncritical.

Set $u_{0}=\emptyset$. Assume that we have constructed in $U$ a sequence $\left(u_{0}, \ldots, u_{m}\right)$ satisfying (1)-(3) for all $k \leq m$. We distinguish the following two alternatives:

(i) Any extension of $u_{m}$ in $U$ is noncritical. In this case we define $n=m$ so that condition (4) is realized, and the construction is finished.

(ii) There exists a critical extension of $u_{m}$ in $U$. In this case we fix such an extension $v$ of minimal length, and define $u_{m+1}=\mu(v)$. Thus $\sigma\left(u_{m+1}\right)=$ $\sigma(v)$ is critical and by Lemma 4.5 we have $u_{m} \prec u_{m+1}$. Finally since $v$ was chosen of minimal length there is no critical subposition $w$ between $u_{m}$ and $u_{m+1}$, and since $u_{m}$ is a good position, it is clear that so is $u_{m+1}$. Hence $u_{m+1}$ satisfies (1)-(3).

Notice that in (ii) one can repeat again the previous arguments starting from $\left(u_{0}, \ldots, u_{m}, u_{m+1}\right)$. If when applying this procedure inductively alternative (i) never occurs then we would obtain an infinite sequence $\left(u_{k}\right)_{k \in \omega}$ satisfying (3) for all $k$, in particular we would have $\mathbf{k}\left(\sigma\left(u_{k-1}\right)\right)<\mathbf{k}\left(\sigma\left(u_{k}\right)\right)$ for all $k$. This clearly defines an infinite run in the game in which the real $y$ constructed by Player I satisfies $\lim \mathbf{k}\left(\left.y\right|_{n}\right)=\infty$, hence $y$ is not in $Y$, and this contradicts the assumption of the lemma. Thus after finitely many steps alternative (i) occurs, and this finishes the construction.

Set $u=u_{n}$ and $p=|u|$, and let $r$ denote the finite run determined by $u$. Fix some $\gamma \in \omega_{1}^{\omega}$ such that $(z, \gamma) \in[T]$ and consider the unique infinite run $(y, z, \theta)$ compatible with $\sigma$, extending $r$ with $\theta(p+n)=\left.\gamma\right|_{n}$ for all $n \geq 0$. It follows easily from (3) and (4) that rule $\left(\mathrm{R}_{1}^{\prime}\right)$ is satisfied, and since $(z, \gamma) \in[T]$, rule $\left(\mathrm{R}_{2}^{\prime}\right)$ is also satisfied. Finally notice that it follows from (2) and (4) that any extension of $u$ in $U$ is again a good position; in particular for all $n$ we have $\left.y\right|_{n+1}=\sigma\left(\left.z\right|_{n},\left.\theta\right|_{n}\right) \in S$, and so $y \in[S]=K$.

Proof of Theorem 4.1. By Lemma 4.4 it is enough to prove that Player II has a winning strategy in the game $G^{\prime}$. So suppose otherwise. Since the game is clearly Borel and so determined, Player I has a winning strategy $\sigma$. Let 
$K$ be the compact set defined above; then by Lemma 4.6, $K$ is a compact subset of $Y$, and by hypothesis (b) of the theorem applied to $K$ we can find $z \in Z$ such that $K \times\{z\} \subset R$. Applying Lemma 4.7 to this $z$ we obtain an infinite run $(y, z, \theta)$ compatible with $\sigma$ and such that $y \in K$; hence $(y, z) \in R$ and Player II wins this run, and this is impossible since $\sigma$ is winning.

\section{Continuous liftings over $\Pi_{3}^{0}$ spaces}

5.1. Theorem. Assume that " $\forall \alpha \in \omega^{\omega}, \omega_{1}^{L(\alpha)}<\omega_{1}$ ". Let $R$ and $Y \times Z$ be two subsets of $2^{\omega} \times 2^{\omega}$ satisfying:

(a) $Y$ is $\boldsymbol{\Pi}_{3}^{0}, Z$ is $\boldsymbol{\Sigma}_{2}^{1}, R$ is $\boldsymbol{\Delta}_{1}^{1}$,

(b) for every compact $K \subset Y$, there exists $z \in Z$ such that $K \times\{z\} \subset R$.

Then there exists a continuous mapping $f: 2^{\omega} \rightarrow 2^{\omega}$ such that $\left.f\right|_{Y} \subset$ $R \cap(Y \times Z)$ where $\left.f\right|_{Y}$ denotes the graph of the restriction of $f$ to $Y$.

As in Theorem 4.1, the proof is easy if $Z$ is Borel. But in the general case we have to keep the game Borel.

Precisely we shall prove the following:

5.2. TheOrem. Fix $\alpha \in \omega^{\omega}$, and assume that " $\omega_{1}^{L(\alpha)}<\omega_{1}$ ". Let $R$ and $Y \times Z$ be two subsets of $2^{\omega} \times 2^{\omega}$ satisfying:

(a) $Y$ is $\Pi_{3}^{0}(\alpha), Z$ is $\Sigma_{2}^{1}(\alpha), R$ is $\Delta_{1}^{1}(\alpha)$,

(b) for every compact $K \subset Y$, there exists $z \in Z$ such that $K \times\{z\} \subset R$.

Then there exists a continuous mapping $f: 2^{\omega} \rightarrow 2^{\omega}$ such that $\left.f\right|_{Y} \subset$ $R \cap(Y \times Z)$.

Warning. All the rest of this section is devoted to the proof of Theorem 5.2. For simplicity we give the proof for $\alpha=0$; the reader can easily check that all the arguments are uniform. The proof follows a scheme completely similar to the proof of Theorem 4.1: We introduce some adapted game $G^{\prime \prime}$, and as in the $\boldsymbol{\Sigma}_{2}^{0}$ case we derive from some given strategy $\sigma$ for Player I in the game a compact set $K$ with the desired properties. Here also the definition of $K$ is based on the analysis of the strategy $\sigma$; and for this we need to consider some particular classes of positions in the game $G^{\prime \prime}$.

We use the same terminology as in 4.1 and define "minimal positions", "good positions", etc. But we warn the reader that the content of these notions will here be different from the previous case. In fact since the rules of the games are different the comparison of two such notions is meaningless. The reason for this choice is first a matter of simplicity, but also to emphasize the common structure of the proofs for Theorem 4.1 and Theorem 5.1. We also introduce a new notion of "very good positions" which has no analog in the previous case, and which heavily relies on the assumption " $\omega_{1}^{L}<\omega_{1}$ ". 
5.3. Index of an $\omega^{2}$-sequence of trees. Let $\mathbf{S}=\left(S_{k}^{j}\right)_{(j, k) \in \omega \times \omega}$ be an $\omega^{2}$ sequence of trees on $\{0,1\}$. As in 4.2 we denote for each $j$ by $\mathbf{k}^{(j)}$ the index of the $\omega$-sequence $\left(S_{k}^{j}\right)_{k \in \omega}$; thus $\mathbf{k}^{(j)}: \operatorname{Seq}(2) \rightarrow \omega$ with

$$
\mathbf{k}^{(j)}(s)= \begin{cases}\min \left\{k<|s|: s \in S_{k}^{j}\right\} & \text { if there is } k<|s| \text { such that } s \in S_{k}^{j}, \\ |s| & \text { if not. }\end{cases}
$$

The index of $\mathbf{S}$ is the mapping $\overline{\mathbf{k}}: \operatorname{Seq}(2) \rightarrow \operatorname{Seq}(\omega)$ defined inductively as follows: $\overline{\mathbf{k}}(\emptyset)=\emptyset$ and for $s \neq \emptyset$ if $\overline{\mathbf{k}}\left(s^{*}\right)$ is defined with $p=\left|\overline{\mathbf{k}}\left(s^{*}\right)\right|$, set $m=\min \left\{j \leq p: \mathbf{k}^{(\mathbf{j})}(\mathbf{s})>\mathbf{k}^{(\mathbf{j})}\left(\mathbf{s}^{*}\right)\right\}$ if $\mathbf{k}^{(\mathbf{j})}(\mathbf{s})>\mathbf{k}^{(\mathbf{j})}\left(\mathbf{s}^{*}\right)$ for some $j \leq p$ and $m=p$ if not, and define

$$
\overline{\mathbf{k}}(s)= \begin{cases}\left.\overline{\mathbf{k}}\left(s^{*}\right)\right|_{m} & \text { if } m<p, \\ \overline{\mathbf{k}}\left(s^{*}\right)^{\sim} \mathbf{k}^{(\mathbf{p})}(\mathbf{s}) & \text { if } m \geq p .\end{cases}
$$

Notice that always either $\overline{\mathbf{k}}(s) \prec \overline{\mathbf{k}}\left(s^{*}\right)$ or $\overline{\mathbf{k}}(s) \succ \overline{\mathbf{k}}\left(s^{*}\right)$; moreover in the last case $|\overline{\mathbf{k}}(s)|=\left|\overline{\mathbf{k}}\left(s^{*}\right)\right|+1$. Since $\overline{\mathbf{k}}(\emptyset)=\emptyset$ it follows that $|\overline{\mathbf{k}}(s)| \leq|s|$ for all $s$.

5.4. Lemma. For any $x \in 2^{\omega}$ we have

$$
x \in \bigcap_{j} \bigcup_{k}\left[S_{k}^{j}\right] \Leftrightarrow \lim _{n}\left|\overline{\mathbf{k}}\left(\left.x\right|_{n}\right)\right|=\infty .
$$

Proof. Set $X=\bigcap_{j} \bigcup_{k}\left[S_{k}^{j}\right]$ and assume $x \in X$; then there exists $\alpha \in \omega^{\omega}$ such that $x \in\left[S_{\alpha(j)}^{j}\right]$ for all $j$. We shall construct inductively $\beta \in \omega^{\omega}$ and an increasing sequence $n_{j}$ of integers such that

$$
\forall n>n_{j},\left.\quad \beta\right|_{j} \preceq \overline{\mathbf{k}}\left(\left.x\right|_{n}\right),
$$

which will show that $\lim _{n \rightarrow \infty}\left|\overline{\mathbf{k}}\left(\left.x\right|_{n}\right)\right|=\infty$.

Set $n_{0}=0$ and notice that $\left.\beta\right|_{0}=\emptyset$. Assume $n_{j}$ and $\left.\beta\right|_{j}$ are already defined. Since $x \in\left[S_{\alpha(j)}^{j}\right]$, the sequence $\left(\mathbf{k}^{(j)}\left(\left.x\right|_{n}\right)\right)_{n}$ is nondecreasing and bounded by $\alpha(j)$, so it is eventually constant. Hence there exist $n_{j+1}>n_{j}$ and $\beta(j) \leq \alpha(j)$ such that $\mathbf{k}^{(j)}\left(\left.x\right|_{n}\right)=\beta(j)$ for $n \geq n_{j+1}$. Then for any $n>n_{j+1}$ and $s=\left.x\right|_{n}$ we have

$$
\left.\beta\right|_{j} \preceq \overline{\mathbf{k}}(s),\left.\quad \beta\right|_{j} \preceq \overline{\mathbf{k}}\left(s^{*}\right), \quad \mathbf{k}^{(j)}(s)=\mathbf{k}^{(j)}\left(s^{*}\right)=\beta(j) .
$$

It now follows from the definition of $\overline{\mathbf{k}}$ that $\left.\beta\right|_{j} \preceq \overline{\mathbf{k}}(s)$, which completes the inductive construction.

Conversely, assume that $\lim _{n \rightarrow \infty}\left|\overline{\mathbf{k}}\left(\left.x\right|_{n}\right)\right|=\infty$. For all $j$, there exists $n_{j}$ such that $\left|\overline{\mathbf{k}}\left(\left.x\right|_{n}\right)\right| \geq j+1$ for all $n \geq n_{j}$. Then by definition of $\overline{\mathbf{k}}(s)$ we have

$$
\left.\overline{\mathbf{k}}\left(\left.x\right|_{n}\right)\right|_{j+1}=\left.\overline{\mathbf{k}}\left(\left.x\right|_{n_{j}}\right)\right|_{j+1}
$$

for $n \geq n_{j}$, hence $\left.x\right|_{n} \in S_{k}^{j}$ for $k=\overline{\mathbf{k}}\left(\left.x\right|_{n_{j}}\right)(j)$, and thus $x \in \bigcup_{k \in \omega}\left[S_{k}^{j}\right]$. Since this holds for every $j$, we have $x \in X$. 
5.5. The game $G^{\prime \prime}$. As for the game $G^{\prime}$, we fix a tree $T$ on $\{0,1\} \times \omega_{1}$ such that $Z$ is the projection of $[T]$ on the first factor. We also fix an $\omega^{2}$ sequence $\mathbf{S}=\left(S_{k}^{j}\right)$ of trees on $\{0,1\}$ such that $Y=\bigcap_{j} \bigcup_{k}\left[S_{k}^{j}\right]$ and denote by $\overline{\mathbf{k}}$ the associated index. Since $Z$ and $Y$ are lightface, we can choose $\mathbf{S}$ to be recursive and $T$ in $L$ (see [7], p. 537).

Let $G^{\prime \prime}$ be the game of type $\Gamma(T)$ in which rule $\left(\mathrm{R}_{2}\right)$ is unchanged and $\left(\mathrm{R}_{1}\right)$ is replaced by

$\left(\mathrm{R}_{1}^{\prime \prime}\right) \quad$ If $(s, t, \theta)$ is any finite run with $|s|=|t|=|\theta|=n+1$ then

$$
\begin{cases}\theta(n)=\left.\theta(n-1)\right|_{m} & \text { if } m=|\overline{\mathbf{k}}(s)| \leq|\theta(n-1)|, \\ \theta(n) \succeq \theta(n-1) \text { and }|\theta(n)| \leq|\theta(n-1)|+1 & \text { if not. }\end{cases}
$$

It is easy to check that in such a run (in which Player II makes the last move) we have $|\theta(n)| \leq|\overline{\mathbf{k}}(s)|$. Formally, the comparison of $\left(\mathrm{R}_{1}^{\prime \prime}\right)$ and $\left(\mathrm{R}_{1}^{\prime}\right)$ is meaningless. However one should think intuitively of $\left(\mathrm{R}_{1}^{\prime \prime}\right)$ as being less restrictive than $\left(\mathrm{R}_{1}^{\prime}\right)$ : one of the main possibilities offered to Player II by $\left(\mathrm{R}_{1}^{\prime \prime}\right)$ (and totally forbidden by $\left(\mathrm{R}_{1}^{\prime}\right)$ ) is to "pass" for the ordinal move at any time by playing $\theta(n)=\theta(n-1)$. This novelty will play a major role in the arguments.

Win condition. Player II wins the infinite run $(y, z, \theta)$ in $G^{\prime \prime}$ if $y \notin Y \quad$ or $\quad\left[(y, z) \in R\right.$ and $\lim _{n} \theta(n)$ exists in $\left.\omega_{1}^{\omega}\right]$.

All game-theoretical notions considered in this section are relative to the game $G^{\prime \prime}$.

5.6. LEMmA. If Player II has a winning strategy then there exists a continuous mapping $f: 2^{\omega} \rightarrow 2^{\omega}$ such that $\left.f\right|_{Y} \subset R \cap(Y \times Z)$.

Proof. Notice that if an infinite run $(y, z, \theta)$ in the game is won by Player II with $y \in Y$, then $\gamma=\lim _{n} \theta(n) \in \omega_{1}^{\omega}$ exists and it follows from rule $\left(\mathrm{R}_{2}\right)$ that $(z, \gamma) \in[T]$.

The rest of the argument is completely similar to Lemma 4.4: A winning strategy for Player II in this game defines canonically a continuous function $f: 2^{\omega} \rightarrow 2^{\omega}$ such that $\left.f\right|_{Y} \subset R \cap(Y \times Z)$.

5.7. Lemma. For any infinite run $(y, z, \theta)$ in the game, the following are equivalent:

(i) $\lim _{n} \theta(n)$ exists in $\omega_{1}^{\omega}$,

(ii) $\lim _{n}|\theta(n)|=\infty$,

(iii) $y \in Y$ and the sequence $(|\theta(n)|)_{n \in \omega}$ of integers is not stationary.

Proof. (iii) $\Rightarrow$ (ii). Define $\alpha$ and $\beta \in \omega^{\omega}$ by $\alpha(n)=\left|\overline{\mathbf{k}}\left(\left.y\right|_{n}\right)\right|$ and $\beta(n)=$ $|\theta(n)|$. It follows from $\left(\mathrm{R}_{1}^{\prime \prime}\right)$ that for all $n$ : 
(1) $\beta(n) \leq \alpha(n)$,

(2) $\beta(n)<\beta(n-1) \Rightarrow \beta(n)=\alpha(n)$.

Set $M=\{n>0: \beta(n)<\beta(n-1)\}$; we distinguish two cases:

CASE 1: $M$ is finite. In this case the sequence $\beta$ of integers is eventually nondecreasing, and since it is not stationary, we have $\lim _{n} \beta(n)=\infty$.

CASE 2: $M$ is infinite. Since $y \in Y$, by Lemma 5.4 in this case we have $\lim _{n} \alpha(n)=\infty$; and we now show that $\lim _{n} \beta(n)=\infty$.

Fix an arbitrary $p$ and then fix $n_{0}$ such that $\alpha(n) \geq p$ for all $n \geq n_{0}$. It follows from condition (2) that for any $m \in M$ with $m \geq n_{0}$ we have $\beta(m)=\alpha(m) \geq p$; moreover if $m^{\prime}$ denotes the first element of $M$ greater than $m$ then on the interval $\left[m, m^{\prime}\right.$ [ the sequence $\beta$ is nondecreasing, hence for all $n \in\left[m, m^{\prime}\right.$ [ we have $\beta(n) \geq \beta(m) \geq p$. It follows that if we fix any $m_{0} \in M$ with $m_{0} \geq n_{0}$ then $\beta(n) \geq p$ for all $n \geq m_{0}$. This proves (ii).

(ii) $\Rightarrow\left(\right.$ i). Fix an infinite sequence $\left(n_{k}\right)$ such that $|\theta(n)| \geq k$ for all $n \geq n_{k}$. It follows from $\left(\mathrm{R}_{1}^{\prime \prime}\right)$ that for all $m, n \geq n_{k}$ we necessarily have $\left.\theta(n)\right|_{k}=$ $\left.\theta(m)\right|_{k}$, which proves (i).

(i) $\Rightarrow$ (iii). If $\lim _{n} \theta(n)$ exists in $\omega_{1}^{\omega}$ then necessarily $\lim _{n}|\theta(n)|=\infty$, hence $y \in Y$ by Lemma 5.4 ; and obviously $(|\theta(n)|)_{n \in \omega}$ is not stationary.

The win condition clearly defines a Borel subset of $2^{\omega} \times 2^{\omega} \times \Sigma^{\omega}$ and so the game $G^{\prime \prime}$ is determined. Moreover we have the following absoluteness property:

5.8. LEMMA. The winning player in the game $G^{\prime \prime}$ has a winning strategy in $L$.

Proof. Consider the game $\widetilde{G}$ in which, at each of their moves, Player I chooses as in $G^{\prime \prime}$ an element in $\{0,1\}$, whereas Player II chooses an element in $\omega \times\{0,1\} \times \Sigma$. Similarly to $G^{\prime \prime}$ identify a run in $\widetilde{G}$ with a quadruple $(r, s, t, \theta)$. The rules in $\widetilde{G}$ are $\left(\mathrm{R}_{1}^{\prime \prime}\right),\left(\mathrm{R}_{2}\right)$ and the additional trivial rule:

$$
r(n)=|\theta(n)| .
$$

Also we identify an infinite run in $\widetilde{\Gamma}$ with a quadruple $(x, y, z, \theta) \in \omega^{\omega} \times$ $2^{\omega} \times 2^{\omega} \times \Sigma^{\omega}$; and Player II wins this run if

$$
y \notin Y \quad \text { or } \quad\left[(y, z) \in R \text { and } \lim _{n} x(n)=\infty\right] .
$$

By Lemma 5.7 the games $G^{\prime \prime}$ and $\widetilde{G}$ are equivalent. Moreover any winning strategy $\widetilde{\sigma}$ for one of the players in $\widetilde{G}$ determines a winning strategy $\sigma$ for the same player in $G^{\prime \prime}$, in a trivially definable way; in particular $\sigma \in L(\widetilde{\sigma})$.

Thus it is enough to prove the lemma for the game $\widetilde{G}$ instead of $G^{\prime \prime}$. For this, notice that the set of all infinite runs won by Player II in $\widetilde{G}$ is of the 
form $\left(B \times \Sigma^{\omega}\right) \cap[\mathcal{T}]$ with $B$ a $\Delta_{1}^{1}$ subset of $\omega^{\omega} \times 2^{\omega} \times 2^{\omega}$ and $\mathcal{T}$ a tree in $L$ on $\omega \times 2 \times 2 \times \Sigma$ determined by the rules of the game; then the conclusion of the lemma follows from [3], Proposition 4.3.

We now fix a strategy $\sigma$ for Player I in the game and we suppose that $\sigma \in L$. All runs and positions considered from now on will implicitly be assumed to be compatible with $\sigma$.

Notations. We recall previous notations and fix new ones. If $u=(t, \theta) \in$ $2^{n} \times \Sigma^{n}$ is a position in the game (compatible with $\left.\sigma\right)$ and $(s, t, \theta)$ is the unique run associated to $u$ with $|s|=n+1$ and $|t|=|\theta|=n$, we set:

$$
\begin{aligned}
\sigma(u) & =s \in 2^{n+1}, \\
\pi(u) & =t \in 2^{n}, \\
\lambda(u) & =\theta(n-1) \in \Sigma, \\
\bar{\lambda}(u) & =\langle\theta(0), \ldots, \theta(n-1)\rangle=\theta \in \Sigma^{n}, \\
\bar{\ell}(u) & =\langle|\theta(0)|, \ldots,|\theta(n-1)|\rangle \in \omega^{n}, \\
\ell_{m}(u) & =|\theta(m)| \in \omega \quad \text { for all } m<n, \\
\varphi(u) & =(\sigma(u), \pi(u), \bar{\ell}(u)) .
\end{aligned}
$$

The mapping $\varphi$ will play a fundamental role in the proof. One should think of the triple $\varphi(u)=(\sigma(u), \pi(u), \bar{\ell}(u))$ as a shadow of the run $(\sigma(u)$, $\pi(u), \bar{\lambda}(u))$. To analyze the strategy $\sigma$ we first reduce the information contained in such a run to the shadow $\varphi(u)$ and to the last ordinal move $\lambda(u)$ (instead of $\bar{\lambda}(u)$ ). The main property of the shadow is to admit only countably many possible values, but it still contains some information from the real run. In fact for technical reasons we need to deal with a more precise shadow, to be introduced later, which again admits only countably many possible values but contains more information about the run.

Special extensions. Let $u$ and $v$ two positions with $|u| \leq|v|$ (but we do not suppose that $u \preceq v$ ). We say that $v$ is $\lambda$-compatible with $u$ if

$$
\left|\lambda\left(\left.v\right|_{m}\right)\right| \geq|\lambda(u)| \quad \text { for all } m \in[|u|,|v|] .
$$

If moreover $v$ is an extension of $u$ it follows from rule $\left(\mathrm{R}_{1}^{\prime \prime}\right)$ that the following stronger property holds:

$$
\lambda\left(\left.v\right|_{m}\right) \succeq \lambda(u) \quad \text { for all } m \in[|u|,|v|]
$$

and we then say that $v$ is a $\lambda$-compatible extension of $u$.

We now discuss more particular types of extensions. Let $v$ be an extension of $u$.

We say that $v$ is a monotone extension of $u$ if

$$
\left.\left.\lambda\left(\left.v\right|_{m}\right) \succeq \lambda\left(\left.v\right|_{m-1}\right) \quad \text { for all } m \in\right]|u|,|v|\right],
$$

that is, $\left.v\right|_{m}$ is $\lambda$-compatible with $\left.v\right|_{m-1}$ for all $\left.\left.m \in\right]|u|,|v|\right]$. 
We say that $v$ is a trivial extension of $u$ if

$$
\left.\left.\lambda\left(\left.v\right|_{m}\right)=\lambda(u) \quad \text { for all } m \in\right]|u|,|v|\right] .
$$

Minimal positions. We shall say that a position $u$ is minimal if for any position $v$ the following implication holds:

$$
(\varphi(v)=\varphi(u) \text { and } \lambda(v)=\lambda(u)) \Rightarrow \bar{\lambda}(u) \leq \bar{\lambda}(v)
$$

where as always $\leq$ refers to the canonical lexicographical ordering on $\operatorname{Seq}(\Sigma)$.

Again given any position $v$ it is clear that there exists a unique minimal position $u$ with $\varphi(v)=\varphi(u)$ and $\lambda(v)=\lambda(u)$, and we shall denote by $\mu(v)$ this unique position $u$.

The $\mu$ operator gives a simple way to construct minimal positions; we now give another (more constructive) way to do this:

5.9. LEMMA. If $u$ is a minimal position and $v$ is a monotone extension of $u$ then $v$ is minimal.

Proof. Let $v^{\prime}$ be a position with $\varphi\left(v^{\prime}\right)=\varphi(v)$ and $\lambda\left(v^{\prime}\right)=\lambda(v)$ and denote by $u^{\prime}$ the restriction of $v^{\prime}$ to $|u|$. Obviously $\varphi\left(u^{\prime}\right)=\varphi(u)$. Moreover since $v$ is a monotone extension of $u$, it follows that $v^{\prime}$ is also a monotone extension of $u^{\prime}$; hence $\lambda\left(u^{\prime}\right)=\lambda(u)$ and by the minimality of $u$ we have $\bar{\lambda}(u) \leq \bar{\lambda}\left(u^{\prime}\right)$ and a fortiori $\bar{\lambda}(v) \leq \bar{\lambda}\left(v^{\prime}\right)$; this proves that $v$ is minimal.

The equivalence relation $\equiv$ on $\Sigma$. We recall that the shadow of a position $u$ is a triple

$$
\varphi(u) \in \operatorname{Seq}(2) \times \operatorname{Seq}(2) \times \operatorname{Seq}(\omega) .
$$

We now define a more precise shadow which will be a quadruple

$$
\widetilde{\varphi}(u)=(\varphi(u), \varepsilon(u)) \in \operatorname{Seq}(2) \times \operatorname{Seq}(2) \times \operatorname{Seq}(\omega) \times \operatorname{Seq}(2)
$$

where $\varepsilon(u)$ is the characteristic function of the set $\left\{m \leq|u|:\left.u\right|_{m}\right.$ is a minimal position\}.

Now for any $\varrho \in \Sigma$ set

$$
\Phi(\varrho)=\{\widetilde{\varphi}(u): u \text { a position with } \lambda(u)=\varrho\}
$$

and define the equivalence relation $\equiv$ on $\Sigma$ by

$$
\varrho \equiv \varrho^{\prime} \Leftrightarrow \Phi(\varrho)=\Phi\left(\varrho^{\prime}\right) .
$$

5.10. Lemma. If $\omega_{1}^{L}<\omega_{1}$ then there mapping $\mathbf{N}: \Sigma \rightarrow \omega$ satisfying:

$\left(\mathrm{N}_{0}\right) \quad \mathbf{N}(\emptyset)=0$,

$\left(\mathrm{N}_{1}\right) \quad \mathbf{N}(\varrho)=\mathbf{N}\left(\varrho^{\prime}\right) \Rightarrow \varrho \equiv \varrho^{\prime}$,

$\left(\mathrm{N}_{2}\right) \quad \varrho \prec \varrho^{\prime} \Rightarrow \mathbf{N}(\varrho) \leq \mathbf{N}\left(\varrho^{\prime}\right)$. 
Proof. Set $E=\operatorname{Seq}(2) \times \operatorname{Seq}(2) \times \operatorname{Seq}(\omega) \times \operatorname{Seq}(2)$. For any $\varrho \in \Sigma$, the set $\Phi(\varrho)$ is a subset of $E$; and this defines a mapping $\Phi: \Sigma \rightarrow \mathcal{P}(E)$.

We recall that the strategy $\sigma$ and the tree $T$ were chosen in $L$. Moreover all the parameters $\mathbf{S}, \overline{\mathbf{k}}, \ldots$ implicitly involved in the definition of $\varphi$ are also in $L$ since they are recursive; and since the lexicographical ordering of $\Sigma$ is in $L$, the relation " $\left.u\right|_{m}$ is a minimal position" (with argument $(u, m)$ ) is also in $L$. Hence the function $\Phi$ is definable in $L$, and $\Phi(\lambda) \in L$ for all $\lambda \in \Sigma$.

On the other hand the set $E$ is countable in $L$, and if we assume that $\omega_{1}^{L}<\omega_{1}$ then the set $\mathcal{P}(E) \cap L$ is countable in $V$. This shows that the range of $\Phi$ is countable in $V$; hence the equivalence relation $\equiv$ on $\Sigma$ has countably many classes. The existence of the required mapping $\mathbf{N}$ is then straightforward.

From now on we assume that $\omega_{1}^{L}<\omega_{1}$ and fix a mapping $\mathbf{N}: \Sigma \rightarrow \omega$ with properties $\left(\mathrm{N}_{0}\right)-\left(\mathrm{N}_{2}\right)$ above.

Pseudo-extension. Let $u$ and $v$ be two minimal positions; we shall say that $v$ is is a pseudo-extension of $u$, and write $u \triangleleft v$, if there exists an extension $v^{\prime}$ of $u$ with $v=\mu\left(v^{\prime}\right)$; such a position $v^{\prime}$ is not unique.

It is fundamental to observe that on the set of all minimal positions the relation $\triangleleft$ is not transitive. However, as we shall see, it has enough transitive consequences.

5.11. Lemma. Let $u$ and $v$ be two minimal positions.

(a) If $u \prec v$ then $u \triangleleft v$.

(b) If $u \triangleleft v$ and $u^{\prime} \prec u$ is minimal, then $u^{\prime} \triangleleft v$.

(c) If $u \triangleleft v$ then $\varphi(u) \prec \varphi(v)$.

(d) If $u \triangleleft v$ and $m \leq|u|$ then $\bar{\lambda}\left(\left.v\right|_{m}\right) \leq \bar{\lambda}\left(\left.u\right|_{m}\right)$.

Proof. (a) and (b) are straightforward. For (c) and (d) fix $v^{\prime}$ such that $v=\mu\left(v^{\prime}\right)$ and $u \prec v^{\prime}$; notice that $\varphi(u) \prec \varphi\left(v^{\prime}\right)=\varphi(v)$ and $\bar{\lambda}(v) \leq \bar{\lambda}\left(v^{\prime}\right)$, hence

$$
\bar{\lambda}\left(\left.v\right|_{m}\right)=\left.\bar{\lambda}(v)\right|_{m} \leq\left.\bar{\lambda}\left(v^{\prime}\right)\right|_{m}=\left.\bar{\lambda}(u)\right|_{m}=\bar{\lambda}\left(\left.u\right|_{m}\right) .
$$

More fundamental is the following converse of property (a) above.

5.12. Lemma. Let $u$ and $v$ be two minimal $\lambda$-compatible positions. If $u \triangleleft v$ then $u \prec v$.

Proof. As above fix $v^{\prime}$ such that $v=\mu\left(v^{\prime}\right)$ and $u \prec v^{\prime}$, and set $m=|u|$. Then $\varphi(u)=\varphi\left(\left.v\right|_{m}\right)$; and since $\bar{\ell}(v)=\bar{\ell}\left(v^{\prime}\right)$, it follows that $v^{\prime}$ is a $\lambda$ compatible extension of $u$ and $\lambda(u)=\left.\lambda\left(v^{\prime}\right)\right|_{m}=\left.\lambda(v)\right|_{m}=\lambda\left(\left.v\right|_{m}\right)$. Then by the minimality of $u$ we have $\bar{\lambda}(u) \leq \bar{\lambda}\left(\left.v\right|_{m}\right)$ and a fortiori $\bar{\lambda}\left(v^{\prime}\right) \leq \bar{\lambda}(v)$; and the minimality of $v$ shows that $v^{\prime}=v$, hence $u \prec v$. 
Good and very good chains. We say that a sequence $\bar{u}=\left(u^{(0)}, \ldots, u^{(m)}\right.$, $\left.\ldots, u^{(n)}\right)$ is a good chain of positions if for all $m \leq n$ :

(1) $u^{(m)}$ is a minimal position,

(2) $\left|u^{(m)}\right|=\left|u^{(m-1)}\right|+1$,

(3) $u^{(m-1)} \triangleleft u^{(m)}$;

and we say that $\bar{u}$ is a very good chain if moreover

(4) $\mathbf{N}\left(\lambda\left(u^{(m)}\right)\right) \leq\left|u^{(m)}\right|$.

Good and very good positions. We shall say that a position $u$ is good (resp. very good) if there exists a good (resp. very good) chain $\bar{u}=\left(u^{(0)}, \ldots\right.$, $\left.u^{(m)}, \ldots, u^{(n)}\right)$ with $u^{(0)}=\emptyset$ and $u^{(n)}=u$.

Such a chain $\bar{u}$ is not unique. By condition (2) for each $m$ we have $\left|u^{(m)}\right|=m$ and obviously each $u^{(m)}$ is then also a good (resp. very good) position. We say that $u^{(m)}$ is a good (resp. very good) minimal version for $\left.u\right|_{m}$. Notice that by Lemma 5.11(c), $\varphi\left(\left.u\right|_{m}\right)=\varphi\left(u^{(m)}\right)$, but $\lambda\left(\left.u\right|_{m}\right)$ and $\lambda\left(u^{(m)}\right)$ might be different, and therefore $u^{(m)}$ is not equal to $\mu\left(\left.u\right|_{m}\right)$ in general. Still we shall see that this will be the case in many situations.

5.13. Examples. (a) By property $\left(\mathrm{N}_{0}\right)$ of Lemma 5.10 the empty sequence $\emptyset$ is a very good position.

(b) If $u$ is any minimal position and $v$ is a monotone extension of $u$, then it follows from Lemmas 5.9 and 5.11(a) that the sequence

$$
\bar{v}=\left(\left.v\right|_{m}\right)_{|u| \leq m \leq|v|}
$$

is a good chain of positions. Hence if $u$ is a good position then so is $v$.

Moreover if $u$ is a very good position and $v$ is a trivial extension of $u$ then it is clear that condition (4) is fulfilled and $\bar{v}$ is a very good chain, hence $v$ is a very good position.

We now come to the crucial property of good positions. To evaluate this property, recall that the restriction of a minimal (and even of a good) position is not minimal in general.

5.14. Lemma. Let $v$ be a (very) good position. If $v$ is a $\lambda$-compatible extension of $u$ then $u$ is a (very) good position; in particular $u$ is minimal.

Proof. Set $m=|u|$ and fix a good chain $\left(v^{(k)}\right)_{k \leq n}$ with $v^{(0)}=\emptyset$ and $v^{(n)}=v$. We prove by induction on $k \in[m, n]$ that $v^{(m)} \preceq v^{(k)}$.

This is obvious for $k=m$. Suppose that $v^{(m)} \preceq v^{(k)}$; since $v^{(k)} \triangleleft$ $v^{(k+1)}$, Lemma 5.11(b), (c) yields $v^{(m)} \triangleleft v^{(k+1)}$ and $\varphi\left(v^{(m)}\right) \prec \varphi\left(v^{(k+1)}\right)$. In particular $v^{(k+1)}$ is $\lambda$-compatible with $v^{(m)}$, hence $v^{(m)} \prec v^{(k+1)}$ by Lemma 5.12 . 
In particular $v^{(m)} \prec v^{(n)}=v$, and since $\left|v^{(m)}\right|=m$, we have $v^{(m)}=$ $\left.v\right|_{m}=u$ and $u$ is a good position. Moreover if $v$ is a very good position then so is $u=\left.v\right|_{m}$.

Definition of the compact set $K$. Let $u$ be a very good position. We recall that the restriction $\left.u\right|_{m}$ of $u$ is not a good position in general. However if $u^{(m)}$ is a very good version of $\left.u\right|_{m}$ (in some very good chain) then $\sigma\left(\left.u\right|_{m}\right)=$ $\sigma\left(u^{(m)}\right) \preceq \sigma(u)$. It follows that the set

$$
S=\{\sigma(u): u \text { a very good position }\} \cup\{\emptyset\}
$$

is a tree on $\{0,1\}$ and we can define the compact set

$$
K=[S] \text {. }
$$

5.15. Lemma. If $\sigma$ takes its values in $Y$ then $K \subset Y$.

Proof. Fix $y \in K$. For each $n$, since $s_{n}=\left.y\right|_{n+1} \in S$, fix a very good position $u_{n}=\left(t_{n}, \theta_{n}\right)$ such that $\left|u_{n}\right|=n$ and $s_{n}=\sigma\left(u_{n}\right)$, and set $\alpha(n)=$ $\left|\overline{\mathbf{k}}\left(s_{n}\right)\right|$.

It follows from rule $\left(\mathrm{R}_{1}^{\prime \prime}\right)$ that $\ell_{m}\left(u_{n}\right) \leq \alpha(m)$ for all $m<n$. Hence by Lemma 2.5 we can extract from $\left(u_{n}\right)$ a subsequence $\left(v_{n}\right)$ such that the limit

$$
\lim \left(\sigma\left(u_{n}\right), \pi\left(u_{n}\right), \bar{\ell}\left(u_{n}\right)\right)=(y, z, \beta) \in 2^{\omega} \times 2^{\omega} \times \omega^{\omega}
$$

exists; and by the previous observations we then have $\beta(n) \leq \alpha(n)$ for all $n$.

Suppose that $y \notin Y$. Then by Lemma 5.4 we would have

$$
p=\liminf _{n} \beta(n) \leq \liminf _{n} \alpha(n)<\infty .
$$

Set

$$
m_{0}=\min \{m \in \omega: \beta(m-1)=p \text { and } \forall n \geq m, \beta(n) \geq p\}
$$

and let $\left(m_{n}\right)_{n \in \omega}$ be the increasing enumeration of the set

$$
M=\left\{m \geq m_{0}: \beta(m-1)=p\right\} .
$$

Replacing $\left(v_{n}\right)$ by some subsequence if necessary we can suppose that for all $n$,

$$
\left|v_{n}\right| \geq m_{n} \text { and }\left.\varphi\left(v_{n}\right)\right|_{m_{n}} \prec(y, z, \beta) .
$$

Let $w_{n}$ be a minimal very good version for $\left.v_{n}\right|_{m_{n}}$ and set $\varrho_{n}=\lambda\left(w_{n}\right)$. Then $\bar{\ell}\left(w_{n}\right) \prec \beta$ for all $n$. It follows from the definition of $M$ that for all $k<n$, the position $w_{n}$ is a $\lambda$-compatible extension of $w_{(n, k)}=\left.w_{n}\right|_{m_{k}}$ (the restriction of $w_{n}$ to $\left.m_{k}\right)$, hence by Lemma $5.14, w_{(n, k)}$ is a very good position and since $\lambda\left(w_{(n, k)}\right)=\varrho_{n}$ we have $\mathbf{N}\left(\varrho_{n}\right) \leq m_{k}$.

In particular for $k=0$ we obtain $\mathbf{N}\left(\varrho_{n}\right) \leq m_{0}$, and replacing $\left(v_{n}\right)$ by some subsequence if necessary we can assume that the sequence $\left(\mathbf{N}\left(\varrho_{n}\right)\right)$ is constant; hence by property $\left(\mathrm{N}_{1}\right)$ of $\mathbf{N}$ all the $\varrho_{n}$ 's are equivalent to some 
fixed $\varrho \in \Sigma$. Applying then the definition of the equivalence relation $\equiv$ we can find for all $n$ a position $w_{n}^{\prime}$ with

$$
\widetilde{\varphi}\left(w_{n}^{\prime}\right)=\widetilde{\varphi}\left(w_{n}\right) \quad \text { and } \quad \lambda\left(w_{n}^{\prime}\right)=\varrho .
$$

Again fix $k<n$ and set as above $w_{(n, k)}^{\prime}=\left.w_{n}^{\prime}\right|_{m_{k}}$. Since $w_{(n, k)}$ is minimal, it follows from the equality $\varepsilon\left(w_{n}^{\prime}\right)=\varepsilon\left(w_{n}\right)$ that $w_{(n, k)}^{\prime}$ is also minimal. It also follows from the equality $\varphi\left(w_{n}^{\prime}\right)=\varphi\left(w_{n}\right)$ that

$$
\varphi\left(w_{(n, k)}^{\prime}\right)=\varphi\left(w_{(n, k)}\right)=\varphi\left(w_{k}^{\prime}\right) \quad \text { and } \quad \lambda\left(w_{(n, k)}^{\prime}\right)=\lambda\left(w_{n}^{\prime}\right)=\lambda\left(w_{k}^{\prime}\right)=\varrho .
$$

Hence by the minimality of both positions $w_{(n, k)}^{\prime}$ and $w_{k}^{\prime}$ we conclude that $w_{k}^{\prime}=w_{(n, k)}^{\prime} \prec w_{n}^{\prime}$.

Thus the sequence $\left(w_{n}^{\prime}\right)$ defines a unique infinite run in the game in which the real constructed by Player I is clearly $y$, and this contradicts the hypothesis of the lemma since $y \notin Y$.

5.16. LEMMA. If $\sigma$ takes its values in $Y$ then for any $z \in Z$ there exists an infinite run $(y, z, \theta)$ (compatible with $\sigma$ ) such that $y \in K$ and $\lim _{n} \theta(n)$ exists.

Proof. Fix $z$ in $Z$ and $\gamma \in \Sigma^{\omega}$ such that $(z, \gamma) \in[T]$. Let $V=V_{(z, \gamma)}$ denote the set of all positions $u$ such that $(\pi(u), \lambda(u)) \prec(z, \gamma)$.

We first construct an infinite sequence $\left(u_{k}\right)_{k \in \omega}$ in $V$ satisfying the following conditions for all $k \in \omega$ :

(1) $u_{k}$ is a very good position,

(2) $u_{k-1} \triangleleft u_{k}$

(3) $\left|\lambda\left(u_{k}\right)\right| \neq\left|\lambda\left(u_{k-1}\right)\right|$.

Set $u_{0}=\emptyset \in V$, which obviously satisfies (1). Assume that $v=u_{k-1}$ is a given very good position in $V$; we shall define $w=u_{k} \in V$ satisfying (1)-(3).

Set $m=|v|$ and $p=|\lambda(v)|$. For all $n \geq m$ let $v_{n}$ denote the unique trivial extension of length $n$ of $v$ such that $\pi\left(v_{n}\right)=\left.z\right|_{n}$, which is in $V$ (in particular $\left.v_{m}=v\right)$, and set $q_{n}=\left|\overline{\mathbf{k}}\left(\sigma\left(v_{n}\right)\right)\right|$.

Say that an extension $v^{\prime}$ of $v$ is critical if $\left|\overline{\mathbf{k}}\left(\sigma\left(v^{\prime}\right)\right)\right|<|\lambda(v)|$. We distinguish the following two alternatives (i) and (ii):

(i) Any trivial extension of $v$ in $V$ is noncritical. Since by the definition of $\overline{\mathbf{k}}$ we have $q_{j} \neq q_{j-1}$ for all $j$, we can find $n \geq \mathbf{N}\left(\left.\gamma\right|_{p+1}\right)$ such that $q_{n}>p$. We fix such an $n$ and define

$$
w=v_{n} \frown\left(z(n),\left.\gamma\right|_{p+1}\right),
$$

which is a legal position by rule $\left(\mathrm{R}_{2}^{\prime \prime}\right)$. Notice that by Lemma 5.9 both $v_{n}$ and $w$ are minimal, hence $v_{n} \triangleleft w$.

(ii) There exists a trivial extension of $v$ in $V$ which is critical. Let $n$ be the least $j \geq m$ such that $q_{j}<p$ and set $q=q_{n}$. Then $v_{n} \frown\left(z(n),\left.\gamma\right|_{q}\right)$ is a 
legal position by rule $\left(\mathrm{R}_{2}^{\prime \prime}\right)$; then we define

$$
w=\mu\left(v_{n} \frown\left(z(n),\left.\gamma\right|_{q}\right)\right) .
$$

Notice that again by Lemma $5.9, v_{n}$ is minimal; moreover $w$ is also minimal and $v_{n} \triangleleft w$. By Example 5.13(b) the sequence $\bar{v}=\left(v_{m}, v_{m+1}, \ldots, v_{n-1}, v_{n}\right)$ is a good chain; since $v_{n} \triangleleft w$ with $|w|=\left|v_{n}\right|+1$, also $\bar{w}=\left(v_{m}, v_{m+1}, \ldots\right.$, $\left.v_{n-1}, v_{n}, w\right)$ is a good chain; since $v$ is very good, for all $k \in[m, n]$ we have

$$
\mathbf{N}\left(\lambda\left(\left.w\right|_{k}\right)\right)=\mathbf{N}\left(\left.\gamma\right|_{p}\right) \leq|v| \leq k .
$$

Moreover in case (i) by the choice of the integer $n$ we have

$$
\mathbf{N}(\lambda(w))=\mathbf{N}\left(\left.\gamma\right|_{p+1}\right) \leq n \leq|w|,
$$

and in case (ii) by property $\left(\mathrm{N}_{2}\right)$ of the mapping $\mathbf{N}$ (see Lemma 5.10) we have

$$
\mathbf{N}(\lambda(w))=\mathbf{N}\left(\left.\gamma\right|_{q}\right) \leq \mathbf{N}\left(\left.\gamma\right|_{p}\right) \leq|v| \leq|w| .
$$

Hence in both cases $\bar{w}$ is a very good chain, and since $v=v_{m}$, the first element of the sequence $\bar{w}$, is a very good position, $w$ is also very good. Moreover since $v \prec v_{n} \triangleleft w$, by Lemma 5.11(b) we have $v \triangleleft w$; and clearly $|\lambda(w)| \neq|\lambda(v)|$. This finishes the construction of the sequence $\left(u_{k}\right)$.

It now follows from Lemma 5.11(d) that for all $j<k$ if $n=\left|u_{j}\right|$ then $\bar{\lambda}\left(u_{j}\right) \geq \bar{\lambda}\left(\left.u_{k}\right|_{n}\right)$, hence by Remarks $2.4(\mathrm{~b}),(\mathrm{c}), \lim _{k} \bar{\lambda}\left(u_{k}\right)=\theta \in \Sigma^{\omega}$ exists and $(y, z, \theta)$ is an infinite run in the game compatible with $\sigma$, and it follows from condition (1) that $y \in[S]=K$.

On the other hand Lemma 5.11(c) shows that if $j<k$ and $n<\left|u_{j}\right|<\left|u_{k}\right|$ then $\ell_{n}\left(u_{j}\right)=\ell_{n}\left(u_{k}\right)$, hence $|\theta(n)|=\lim _{k} \ell_{n}\left(u_{k}\right)=\ell_{n}\left(u_{k}\right)$. In particular for $n_{k}=\left|u_{k}\right|-1$ we have $\left|\theta\left(n_{k}\right)\right|=\left|\lambda\left(u_{k}\right)\right|$ and condition (3) yields that the sequence $(|\theta(n)|)_{n}$ is not stationary. Finally since by the hypothesis of the lemma, $\sigma$ takes its values in $Y$, we have $y \in Y$ and it follows from Lemma 5.7 that $\lim _{n} \theta(n)$ exists in $\left(\omega_{1}\right)^{\omega}$.

Proof of Theorem 5.2. The argument is totally similar to 4.1, and we leave the details to the reader. Notice only that by Lemma 5.8 we can choose $\sigma$, the supposed winning strategy for Player I, in $L$.

6. Cofinal embedding of $\Pi_{1}^{1}$-sets in $\mathcal{K}(\mathbb{Q})$. Throughout this section we denote by $\mathbb{Q}$ the countable dense-in-itself subset of $2^{\omega}$ defined by

$$
\mathbb{Q}=\left\{\alpha \in 2^{\omega}: \exists m \forall n>m, \alpha(n)=0\right\} .
$$

We denote by $\mathbf{0}$ the null sequence $(\mathbf{0}(p)=0$ for all $p)$, and for all $n \in \omega$ by $e_{n}$ the characteristic function of $\{n\}\left(e_{n}(p)=1 \Leftrightarrow p=n\right)$; obviously $\mathbf{0}$ and all the $e_{n}$ 's are elements of $\mathbb{Q}$, and $\mathbf{0}=\lim _{n} e_{n}$.

It is a well known and basic fact that the $\Pi_{1}^{1}$ set $\mathcal{K}(\mathbb{Q})$ is $\Pi_{1}^{1}$-complete, in particular non-Borel. Thus for any $\Pi_{1}^{1}$ set $W \subset 2^{\omega}$ there exists a continuous mapping $g: 2^{\omega} \rightarrow \mathcal{K}\left(2^{\omega}\right)$ which reduces $W$ to $\mathcal{K}(\mathbb{Q})$, that is, 
$W=g^{-1}(\mathcal{K}(\mathbb{Q}))$. One can even assume that $g$ is a homeomorphic embedding. In fact if $g: 2^{\omega} \rightarrow \mathcal{K}\left(2^{\omega}\right)$ is any continuous reduction of $W$ to $\mathcal{K}(\mathbb{Q})$ and if we define $h$ from $2^{\omega}$ into $\mathcal{K}\left(\{0,1\} \times 2^{\omega}\right) \approx \mathcal{K}\left(2^{\omega}\right)$ by

$$
h(\alpha)=(\{1\} \times g(\alpha)) \cup\{\mathbf{0}\} \cup\left\{e_{n+1}: \alpha(n)=1\right\}
$$

then one easily checks that $h$ is a homeomorphic embedding which also reduces $W$ to $\mathcal{K}(\mathbb{Q})$. In particular:

6.1. Lemma. Any Borel space is homeomorphic to a relatively closed subset of $\mathcal{K}(\mathbb{Q})$.

But for our purpose we need more: we would like the set $\mathcal{A}=h(W)$ to be cofinal in $\mathcal{K}(\mathbb{Q})$. As mentioned in the introduction, this is impossible if $W$ is Borel, since $\mathcal{K}(\mathbb{Q})$ is not analytic. But even if $W$ is non-Borel one cannot in general ensure the cofinality condition on $\mathcal{A}$. However we shall prove the following substitute:

6.2. Theorem. For any $\Pi_{1}^{1}$ subset $W$ of $2^{\omega}$ there exists a homeomorphism $h: G \rightarrow \mathcal{G}$ from a $\mathbf{G}_{\delta}$ subset $G$ of $2^{\omega} \times \omega^{\omega}$ onto a $\mathbf{G}_{\delta}$ subset $\mathcal{G}$ of $\mathcal{K}\left(2^{\omega}\right)$ satisfying:

(1) $W \times \omega^{\omega} \subset G \subset 2^{\omega} \times \omega^{\omega}$,

(2) $W \times \omega^{\omega}=h^{-1}(\mathcal{K}(\mathbb{Q}))$,

(3) if $W$ is non-Borel then $h\left(W \times \omega^{\omega}\right)$ is cofinal in $\mathcal{K}(\mathbb{Q})$.

We start by some general lemmas, some of which might be well known; we give the proofs for completeness.

6.3. Lemma. Let $E$ be a perfect subset of $2^{\omega}, D$ a countable dense subset of $E$ and $a \in D$. Then there exists a homeomorphism $\varphi$ from $E$ onto $2^{\omega}$ such that $\varphi(D)=\mathbb{Q}$ and $\varphi(a)=\mathbf{0}$.

Proof. Let $\left(a_{n}\right)$ be an enumeration of $D$ with $a_{0}=a$. We construct inductively, for every $s \in 2^{<\omega}$, a nonempty clopen subset $E_{s}$ of $E$ and a point $x_{s} \in E_{s} \cap D$ such that:

(i) $E_{\emptyset}=E$,

(ii) $E_{s}=E_{s \frown 0} \cup E_{s \frown 1}$,

(iii) $E_{s \frown 0} \cap E_{s \frown 1}=\emptyset$,

(iv) $\forall x, y \in E_{s} \forall n<|s|, x(n)=y(n)$,

(v) $x_{\emptyset}=a$,

(vi) $x_{s} \frown 0=x_{s}$,

(vii) $x_{s \frown 1}=a_{n}$ for $n=\min \left\{m: a_{m} \in E_{s \frown 1}\right\}$.

If $E_{s}$ is chosen for $|s|=k-1$, then it is perfect; hence it is possible to partition it into two clopen nonempty subsets $E_{s}^{\prime}$ and $E_{s}^{\prime \prime}$ such that the $k$ th coordinate is constant on each of them. Since $x_{s} \in E_{s}$ we can, up to interchanging $E_{s}^{\prime}$ and $E_{s}^{\prime \prime}$, assume that $x_{s} \in E_{s}^{\prime}$. We then put 
$E_{s \frown 0}=E_{s}^{\prime}, E_{s \frown 1}=E_{s}^{\prime \prime}, x_{s \frown 0}=x_{s}$ and $x_{s \frown 1}=a_{m}$ for the least $m$ such that $a_{m} \in E_{s}^{\prime \prime}$.

Then for every $x \in E$ there exists a unique $\alpha=\varphi(x) \in 2^{\omega}$ such that

$$
\forall s \in 2^{<\omega}, \quad x \in E_{s} \Leftrightarrow s \prec \alpha .
$$

It follows from condition (vi) that $\varphi\left(x_{s}\right)=s \frown \mathbf{0}$ for every $s \in 2^{<\omega}$. The function $\varphi$ is continuous since the $E_{s}$ 's are clopen, and bijective since (ii) and (iii) hold. Thus $\varphi$ is a homeomorphism because $E$ is compact. Moreover $\varphi\left(x_{s}\right)=s \frown \mathbf{0}$, hence $\varphi(D) \supset \mathbb{Q}$.

Finally, let $d$ denote the standard metric on $2^{\omega}: d(\alpha, \beta)=\sup \left\{2^{-n}\right.$ : $\alpha(n) \neq \beta(n)\}$. If $\varphi\left(a_{n}\right) \notin \mathbb{Q}$ and $2^{-k}<\min _{j<n} d\left(a_{n}, a_{j}\right)$, then there exists $s \in 2^{<\omega}$ such that $|s| \geq k$ and $s \frown 1 \prec \varphi\left(a_{n}\right)$. Then by condition (iv) none of the $a_{j}$ 's for $j<n$ belongs to $E_{s \frown 1}$. But since $a_{n} \in E_{s \frown 1}$, we have $x_{s \frown 1}=a_{n}$ and $\varphi\left(a_{n}\right)=s \frown 1 \frown \mathbf{0} \in \mathbb{Q}$. This contradiction completes the proof.

6.4. Lemma. Let $S$ and $T$ be two compact subsets of $\mathbb{Q}$. If $f$ is a homeomorphism from $S$ onto $T$, then there exists a homeomorphism $\tilde{f}$ : $2^{\omega} \rightarrow 2^{\omega}$ extending $f$ such that $\tilde{f}(\mathbb{Q})=\mathbb{Q}$.

Proof. Let $d$ denote as before the standard metric on $2^{\omega}$. If $S$ and $T$ are finite then there exists a finite partition of $2^{\omega}$ into clopen sets each containing exactly one point of $S$ (resp. $T$ ); and it follows from the previous lemma that if $U$ and $V$ are clopen neighborhoods of $a \in S$ and $b \in T$ there exists a homeomorphism $f_{U}$ from $U$ to $V$ such that $f_{U}(a)=b$ and $f_{U}(U \cap \mathbb{Q})=V \cap \mathbb{Q}$.

If $S$ and $T$ are infinite, denote by $S^{\prime}$ and $T^{\prime}$ their Cantor derivatives. Let $\left(a_{n}\right)$ be an enumeration of the set $S \backslash S^{\prime}$ of isolated points of $S$. Since $f$ is a homeomorphism and $d\left(a_{n}, S^{\prime}\right) \rightarrow 0$, we have $T^{\prime}=f\left(S^{\prime}\right)$ and $d\left(f\left(a_{n}\right), T^{\prime}\right) \rightarrow 0$. For $n \in \omega$ define

$$
B_{n}=\left\{x \in 2^{\omega} \backslash S^{\prime}: \inf _{p \in \omega} d\left(x, a_{p}\right)=d\left(x, a_{n}\right)<\inf _{j<n} d\left(x, a_{j}\right)\right\} .
$$

It is easy to see that the $B_{n}$ 's are clopen in $2^{\omega}$ and pairwise disjoint, $a_{n} \in B_{n}$, and $\bigcup_{n} B_{n}=2^{\omega} \backslash S^{\prime}$. Moreover $\operatorname{diam}\left(B_{n}\right) \rightarrow 0$ : if not there would exist $\delta>0$, an increasing sequence $\left(n_{i}\right)$ of integers and a sequence $\left(x_{i}\right)$ in $2^{\omega}$ such that $x_{i} \in B_{n_{i}}$ and $d\left(x_{i}, a_{n_{i}}\right) \geq \delta$. Without loss of generality, one could assume that $\left(x_{i}\right)$ converges to some $x \in 2^{\omega}$. If $x \notin S^{\prime}$, there would be some $m$ such that $x \in B_{m}$, and we would have $x_{i} \in B_{m}$, hence $n_{i}=m$, for $i$ large enough. Thus $x \in S^{\prime}$, and there is some $p$ such that $d\left(x, a_{p}\right)<\delta / 2$; hence for large $i, d\left(x_{i}, a_{p}\right)<\delta / 2$ and $n_{i}>p$. This would imply that

$$
\delta \leq d\left(x_{i}, a_{n_{i}}\right)<\inf _{j<n_{i}} d\left(x_{i}, a_{j}\right) \leq d\left(x_{i}, a_{p}\right)<\delta / 2,
$$

a contradiction. Since $B_{n} \cap \mathbb{Q}$ is countable and dense in the perfect set $B_{n}$, 
there exists by Lemma 6.3 a homeomorphism $\varphi_{n}: B_{n} \rightarrow 2^{\omega}$ such that $\varphi_{n}\left(B_{n} \cap \mathbb{Q}\right)=\mathbb{Q}$ and $\varphi_{n}\left(a_{n}\right)=\mathbf{0}$.

Similarly, the sets

$$
C_{n}=\left\{x \in 2^{\omega} \backslash T^{\prime}: \inf _{p \in \omega} d\left(x, f\left(a_{p}\right)\right)=d\left(x, f\left(a_{n}\right)\right)<\inf _{j<n} d\left(x, f\left(a_{j}\right)\right)\right\}
$$

are clopen in $2^{\omega}$, pairwise disjoint, cover $2^{\omega} \backslash T^{\prime}$ and $\operatorname{diam}\left(C_{n}\right) \rightarrow 0$. Thus there exists a homeomorphism $\psi_{n}: C_{n} \rightarrow 2^{\omega}$ such that $\psi_{n}\left(C_{n} \cap \mathbb{Q}\right)=\mathbb{Q}$ and $\psi_{n}\left(f\left(a_{n}\right)\right)=\mathbf{0}$. Then $f_{n}=\psi_{n}^{-1} \circ \varphi_{n}$ is a homeomorphism from $B_{n}$ onto $C_{n}$ such that $f_{n}\left(B_{n} \cap \mathbb{Q}\right)=C_{n} \cap \mathbb{Q}$ and $f_{n}\left(a_{n}\right)=f\left(a_{n}\right)$.

Then if we define $\tilde{f}$ by

$$
\widetilde{f}(y)= \begin{cases}f(y) & \text { if } y \in S^{\prime} \\ f_{n}(y) & \text { if } y \in B_{n}\end{cases}
$$

it is not hard to check that $\tilde{f}$ is a homeomorphism from $2^{\omega}$ to itself extending $f$, and $\widetilde{f}(\mathbb{Q})=\mathbb{Q}$.

6.5. Lemma. Let $W$ be a coanalytic subset of $2^{\omega}$. Then there exists a continuous function $\varphi: 2^{\omega} \rightarrow \mathcal{K}\left(2^{\omega}\right)$ such that

$$
w \in W \Leftrightarrow \varphi(w) \subset \mathbb{Q} \Leftrightarrow \varphi(w) \text { is countable. }
$$

Proof. Let $\mathcal{T}$ be the set of trees on $\omega$, endowed with the topology induced by $2^{\left(\omega^{<\omega}\right)}$. It is well known that the set $\mathcal{W F}=\{T \in \mathcal{T}: T$ is well founded $\}$ is $\boldsymbol{\Pi}_{1}^{1}$-complete in $\mathcal{T}$. Thus there exists a continuous mapping $w \mapsto T_{w}$ from $2^{\omega}$ to $\mathcal{T}$ such that $w \in W \Leftrightarrow T_{w} \in \mathcal{W} \mathcal{F}$.

For any $s=\left(n_{0}, n_{1}, \ldots, n_{k}\right) \in \omega^{<\omega}$ consider the point $q_{s} \in \mathbb{Q}$ with all coordinates zero except those of indices $n_{0}, n_{0}+n_{1}+1, \ldots, n_{0}+n_{1}+\ldots+$ $n_{k}+k$. Then we put

$$
\varphi_{0}(w)=\overline{\left\{q_{s}: s \in T_{w}\right\}} .
$$

If $w \in W$, then $\varphi_{0}(w) \subset \mathbb{Q}$. Indeed, if $\beta \in \varphi_{0}(w) \backslash \mathbb{Q}$, there is a sequence $\left(s_{i}\right)$ in $T_{w}$ such that $q_{s_{i}} \rightarrow \beta$ and there is an infinite sequence $\alpha=\left(n_{k}\right)$ such that the nonzero coordinates of $\beta$ are those of indices $n_{0}, n_{0}+n_{1}+1, \ldots$, $n_{0}+n_{1}+\ldots+n_{k}+k, \ldots$ For each integer $k$ we have $\alpha_{\mid k} \prec s_{i}$ for $i$ large enough, hence $\alpha_{\mid k} \in T_{w}$; this means that $\alpha \in[T]$, so $T \notin \mathcal{W F}$ and this is a contradiction with $w \in W$.

Conversely, if $w \notin W$, then $T_{w} \notin \mathcal{W F}$ and there exists $\alpha \in[T]$. If $q^{(k)}=\left.q_{\alpha}\right|_{k}$, then $q^{(k)} \in \varphi_{0}(w)$ and the sequence $\left(q^{(k)}\right)$ converges to some point $\beta \in 2^{\omega} \backslash \mathbb{Q}$. Then $\beta \in \varphi_{0}(w) \backslash \mathbb{Q}$ and $\varphi_{0}(w) \not \subset \mathbb{Q}$.

For every open subset $U$ of $2^{\omega}$, if $\varphi_{0}(w) \cap U \neq \emptyset$, there exists $s \in T_{w}$ such that $q_{s} \in U$. Then $N=\left\{y: s \in T_{y}\right\}$ is a clopen neighborhood of $w$, and $\varphi_{0}(y) \cap U \neq \emptyset$ for every $y \in N$; hence $\left\{w \in 2^{\omega}: \varphi_{0}(w) \cap U \neq \emptyset\right\}$ is open.

If $F$ is a closed subset of $2^{\omega}$, we now prove that $Z=\left\{w: \varphi_{0}(w) \cap F \neq \emptyset\right\}$ is closed. Assume $\left(w_{i}\right)$ is a sequence in $Z$ which converges to some $w$. For 
each $i$ we can choose $\beta_{i} \in F \cap \varphi_{0}\left(w_{i}\right)$, and extracting a subsequence if necessary, we assume that $\beta_{i} \rightarrow \beta \in F$. And there are $s_{i} \in T_{w_{i}}$ such that $q_{s_{i}} \rightarrow \beta$.

Then there are two possibilities:

- $\beta \in \mathbb{Q}$ : in this case $\beta=q_{s}$ and $s \prec s_{i}$ for $i$ large enough. This implies that $s \in T_{w_{i}}$, thus $s \in T_{w}=\lim _{i \rightarrow \infty} T_{w_{i}}$; that means $\beta=q_{s} \in \varphi_{0}(w) \cap F$, hence $w \in Z$.

- $\beta \notin \mathbb{Q}$ : in this case there is an infinite sequence $\alpha=\left(n_{k}\right)$ such that the nonzero coordinates of $\beta$ are those of indices $n_{0}, n_{0}+n_{1}+1, \ldots, n_{0}+n_{1}+\ldots+$ $n_{k}+k, \ldots$ For each integer $k$ we have $\left.\alpha\right|_{k} \prec s_{i}$, hence $\left.\alpha\right|_{k} \in T_{w_{i}}$ for $i$ large enough, which means $\left.\alpha\right|_{k} \in T_{w}=\lim _{i \rightarrow \infty} T_{w_{i}}$ and $\beta=\left.\lim _{k \rightarrow \infty} q_{\alpha}\right|_{k} \in F$. Thus $w \in Z$.

This completes the proof of the continuity of $\varphi_{0}: 2^{\omega} \rightarrow \mathcal{K}\left(2^{\omega}\right)$.

We endow $2^{\omega}$ with the order $\leq$ defined by $\beta \leq \beta^{\prime} \Leftrightarrow \forall n, \beta(n) \leq \beta^{\prime}(n)$, and define the mapping $\varphi: 2^{\omega} \rightarrow \mathcal{K}\left(2^{\omega}\right)$ by

$$
\beta \in \varphi(w) \Leftrightarrow \exists \beta^{\prime} \in \varphi_{0}(w), \beta \leq \beta^{\prime} .
$$

Clearly $\varphi(w)$ is a compact subset of $2^{\omega}$ for every $w \in 2^{\omega}$. Moreover if $w \in W$ and $\beta \in \varphi(w)$ then there is $\beta^{\prime} \in \varphi_{0}(w)$ such that $\beta \leq \beta^{\prime}$. Since $\beta^{\prime} \in \mathbb{Q}$, there is some $n$ such that $\beta^{\prime}(p)=0$ for $p>n$; and thus $\beta(p) \leq \beta^{\prime}(p)=0$ for $p>n$. Hence $\beta \in \mathbb{Q}$. This shows that $\varphi(w) \subset \mathbb{Q}$ for $w \in W$.

On the other hand, if $w \notin W$, there exists $\beta^{\prime} \in \varphi_{0}(w) \backslash \mathbb{Q}$. Since $\beta^{\prime}$ has infinitely many nonzero coordinates, $\left\{\beta: \beta \leq \beta^{\prime}\right\}$ is uncountable, and so is $\varphi(w)$.

We conclude that

$$
w \in W \Leftrightarrow \varphi(w) \subset \mathbb{Q} \Leftrightarrow \varphi(w) \text { is countable. }
$$

We now show that $\varphi$ is continuous. Let $F$ be a closed subset of $2^{\omega}$; we want to prove that $Z=\{w: \varphi(w) \cap F \neq \emptyset\}$ is closed. But, if $F^{\prime}$ is the compact set $\left\{\beta^{\prime}: \exists \beta \in F, \beta \leq \beta^{\prime}\right\}$, we have

$$
Z=\left\{w: \varphi_{0}(w) \cap F^{\prime} \neq \emptyset\right\},
$$

which is closed since $\varphi_{0}$ is continuous.

Similarly, if $U$ is an open subset of $2^{\omega}$, it is easily checked that

$$
U^{\prime}=\left\{\beta^{\prime}: \exists \beta \in U, \beta \leq \beta^{\prime}\right\}=\bigcup_{\beta \in U \cap \mathbb{Q}}\left\{\beta^{\prime}: \beta \leq \beta^{\prime}\right\}
$$

is open since $\left\{\beta^{\prime}: \beta \leq \beta^{\prime}\right\}$ is open for $\beta \in \mathbb{Q}$. Hence we deduce as above that $\{w: \varphi(w) \cap U \neq \emptyset\}=\left\{w: \varphi_{0}(w) \cap U^{\prime} \neq \emptyset\right\}$ is open.

6.6. Lemma. There exists a homeomorphic embedding $\Phi: \mathcal{K}(\mathbb{Q}) \times 2^{\omega} \rightarrow$ $\mathcal{K}(\mathbb{Q})$ such that $S \subset \Phi(S, \varepsilon)$ for all $\varepsilon \in 2^{\omega}$ and all $S \in \mathcal{K}(\mathbb{Q})$. 
Proof. This is exactly the same mapping as the one constructed in [3], Fact 6.3.1, although the statements are not the same. We give the simple argument for completeness.

Let $\left(U_{n}\right)$ be an increasing sequence of clopen subsets of $\mathbb{Q}$ which covers $\mathbb{Q}$ (one can assume $U_{0}=\emptyset$ ). For all $n$, choose in $V_{n}=U_{n+1} \backslash U_{n}$ a point $a_{n}$, and a sequence $\left(a_{n, p}\right)_{p}$ converging to $a_{n}$.

For $n \in \omega$ and $\beta \in 2^{\omega}$ define the compact set

$$
K_{n}(\beta)=\left\{a_{n}\right\} \cup\left\{a_{n, p}: \beta(p)=1\right\} .
$$

It is clear that the function $K_{n}$ is continuous from $2^{\omega}$ into $\mathcal{K}(\mathbb{Q})$ and oneto-one; so it is a homeomorphism onto its range. Now define

$$
\Phi(S, \beta)=S \cup K_{n}(\beta) \quad \text { if } S \subset U_{n} \text { and } S \cap V_{n-1} \neq \emptyset .
$$

Let $\mathcal{S}_{n}=\left\{S: S \subset U_{n}\right.$ and $\left.S \cap V_{n-1} \neq \emptyset\right\}$. Since $\left(\mathcal{S}_{n}\right)_{n}$ is a partition of $\mathcal{K}(\mathbb{Q})$ into clopen subsets, we see that $\Phi$ is continuous, one-to-one and satisfies $\Phi(S, \beta) \supset S$.

Furthermore, if $S^{\prime}=\Phi(S, \beta)$ and $n=\min \left\{k: S^{\prime} \subset U_{k+1}\right\}$, we have $S^{\prime} \in \mathcal{S}_{n+1}, S=S^{\prime} \cap U_{n}$ and $K_{n}(\beta)=S^{\prime} \cap V_{n}$; and this shows that $n, S$ and $\beta$ depend continuously on $S^{\prime}$, that is, $\Phi^{-1}$ is continuous on its domain.

Proof of Theorem 6.2. Let $\mathcal{C}$ denote the space of all continuous functions from $2^{\omega}$ into itself equipped with the metric of uniform convergence, and let $H^{\prime}$ be the group of homeomorphisms of $2^{\omega}$ preserving $\mathbb{Q}$. By means of the mapping $f \mapsto\left(f, f^{-1}\right)$ we identify $H^{\prime}$ with the set

$$
H=\{(f, g) \in \mathcal{C} \times \mathcal{C}: f \circ g=g \circ f=\operatorname{Id} \text { and } f(\mathbb{Q})=g(\mathbb{Q})=\mathbb{Q}\} .
$$

One easily checks that $H$ is a $\Pi_{3}^{0}$ subset of the Polish space $\mathcal{C} \times \mathcal{C}$. Moreover $H$ is a topological group acting on $\mathcal{K}(\mathbb{Q})$ and it follows from Lemma 6.4 that $H$ is uncountable since so is the set of images under $H$ of any infinite compact subset of $\mathbb{Q}$. Thus the uncountable topological group $H$ has only condensation points, and by a classical result of Sierpiński [12], it is a oneto-one continuous image of $\omega^{\omega}$.

Fix $\theta: \omega^{\omega} \rightarrow H$ and $\varrho: 2^{\omega} \times 2^{\omega} \rightarrow 2^{\omega}$ with $\theta$ a continuous bijection and $\varrho$ a homeomorphic embedding. For any $(w, \alpha) \in W \times \omega^{\omega}$ with $\theta(\alpha)=$ $(f, g) \in H$ set

$$
h(w, \alpha)=\Phi(g(\varphi(w)), \varrho(w, \alpha))
$$

where $\Phi$ is as in Lemma 6.6 and $\varphi$ as in Lemma 6.5.

Then $h$ clearly defines a one-to-one and continuous mapping from $W \times \omega^{\omega}$ into $\mathcal{K}(\mathbb{Q})$. Moreover if $h(w, \alpha)=S^{\prime}$ then $(g(\varphi(w)), \varrho(w, \alpha))=(S, \beta)=$ $\Phi^{-1}\left(S^{\prime}\right)$ and $(w, \alpha)=\varrho^{-1}(\beta)$, which shows that $h^{-1}$ is continuous on its domain. 
Since $h$ is a homeomorphism from $W \times \omega^{\omega}$ onto its range, it extends to a homeomorphism (which we also denote by $h$ ) from a $\mathbf{G}_{\delta}$ subset $G$ of $2^{\omega} \times \omega^{\omega}$ onto some $\mathbf{G}_{\delta}$ subset $\mathcal{G}$ of $\mathcal{K}\left(2^{\omega}\right)$.

From the definition of $h$ we have immediately $h\left(W \times \omega^{\omega}\right) \subset \mathcal{K}(\mathbb{Q})$. On the other hand notice that for any $(w, \alpha) \in W \times \omega^{\omega}$ with $\theta(\alpha)=(f, g) \in H$ Lemma 6.6 yields $h(w, \alpha) \supset g(\varphi(w))$, hence by the continuity of $h$ this also holds for any $(w, \alpha) \in G$. Thus for any $(w, \alpha) \in G$ with $\theta(\alpha)=(f, g) \in H$, if $w \notin W$ then $\varphi(w) \not \subset \mathbb{Q}$ by Lemma 6.5, and since $\mathbb{Q}$ is invariant under $f$ we have $g(\varphi(w)) \not \subset \mathbb{Q}$, hence by the previous observations also $h(w, \alpha) \not \subset \mathbb{Q}$. Thus we also have the converse inclusion $h\left(G \backslash W \times \omega^{\omega}\right) \subset \mathcal{K}\left(2^{\omega}\right) \backslash \mathcal{K}(\mathbb{Q})$, and this proves $(2)$.

To prove (3) notice first that since the Cantor rank $\gamma$ is a $\Pi_{1}^{1}$-norm on the set $\mathcal{K}_{\omega}\left(2^{\omega}\right)$ of countable compact subsets of $2^{\omega}$, and since $\varphi$ reduces $W$ to $\mathcal{K}_{\omega}\left(2^{\omega}\right)$ by Lemma $6.5, \gamma \circ \varphi$ is also a $\Pi_{1}^{1}$-norm on $W$.

Thus if $W$ is not Borel, this $\boldsymbol{\Pi}_{1}^{1}$-norm is not bounded on $W$ :

$$
\forall \xi<\omega_{1}, \exists w \in W, \quad \gamma \circ \varphi(w)>\xi .
$$

In particular for any $S \in \mathcal{K}(\mathbb{Q})$ there exists $w \in W$ such that $\gamma(\varphi(w))>$ $\gamma(S)$. Hence $S$ is homeomorphic to a compact subset $T$ of $\varphi(w)$, and by Lemma 6.4, there exists a homeomorphism $f$ from $2^{\omega}$ to itself such that $f(\mathbb{Q})=\mathbb{Q}$ and $T=f(S)$. If $\alpha$ is such that $\theta(\alpha)=\left(f, f^{-1}\right)=(f, g) \in H$ then

$$
S=g(T) \subset g(\varphi(w)) \subset h(w, \alpha)=S^{\prime},
$$

which proves (3).

7. Applications. We first state a more complete version of Theorem A which follows from the results proved in [3]:

7.1. ThEOREM. The following are equivalent:

(i) For all $\alpha \in \omega^{\omega}$ the set $\omega^{\omega} \cap L(\alpha)$ is $\leq^{\star}$-bounded in $\omega^{\omega}$.

(ii) Any compact covering mapping from a $\boldsymbol{\Pi}_{1}^{1}$ space onto a $\boldsymbol{\Pi}_{2}^{0}$ space is inductively perfect.

(iii) Any compact covering mapping from a $\boldsymbol{\Pi}_{1}^{1}$ space onto a $\mathbf{P}_{\sigma}$ space is inductively perfect.

(iv) In any $\boldsymbol{\Pi}_{2}^{0}$ space $X$, any $\boldsymbol{\Pi}_{1}^{1}$ cofinal subset of $\mathcal{K}(X)$ is continuously cofinal.

(v) Any $\Pi_{1}^{1}$ cofinal subset of $\mathcal{K}\left(\omega^{\omega}\right)$ admits a Borel domination function.

We now prove our first main result:

7.2. Theorem. The following are equivalent:

(i) For all $\alpha \in \omega^{\omega}$ the set $\omega^{\omega} \cap L(\alpha)$ is countable. 
(ii) Any compact covering mapping from a $\boldsymbol{\Pi}_{3}^{0}$ space (onto any space) is inductively perfect.

(iii) Any compact covering mapping from a $\boldsymbol{\Pi}_{3}^{0}$ space onto a $\mathbf{\Pi}_{3}^{0}$ space is inductively perfect.

(iv) Any compact covering mapping from a $\boldsymbol{\Pi}_{1}^{1}$ space onto a $\boldsymbol{\Pi}_{3}^{0}$ space is inductively perfect.

(v) Any compact covering mapping from a $\boldsymbol{\Pi}_{1}^{1}$ space onto a $\check{\mathbf{P}}_{\sigma}$ space is inductively perfect.

Proof. (ii) $\Rightarrow$ (iii) and (iv) $\Rightarrow(v)$ are obvious.

(i) $\Rightarrow$ (ii). Let $f: X \rightarrow Y$ be a compact covering mapping defined on a $\boldsymbol{\Pi}_{3}^{0}$ space $X$. By standard arguments (see Theorem 3.1 of [2]) one can reduce the general case to the case where $X \subset 2^{\omega} \times 2^{\omega}$ and $Y=\pi(X) \subset 2^{\omega}$, where $\pi$ denotes the canonical projection mapping from $2^{\omega} \times 2^{\omega}$ onto the first factor, and $f=\pi_{X}$ is the restriction of $\pi$ to $X$.

We recall that by [2], Theorem 8.8, under assumption (i), the image under a compact covering mapping of a Borel space is also Borel and of the same additive or multiplicative class; hence $Y$ is also $\boldsymbol{\Pi}_{3}^{0}$. Set

$$
\mathcal{C}=\mathcal{K}\left(2^{\omega} \times 2^{\omega}\right), \quad Z=\mathcal{K}(X) \subset \mathcal{C}, \quad R=\left\{(y, H) \in 2^{\omega} \times \mathcal{C}: y \in \pi(H)\right\} .
$$

Then clearly $R$ is a closed subset of $2^{\omega} \times \mathcal{C}$, and $Z$ is $\Pi_{1}^{1}$. Identifying then $\mathcal{C}$ with $2^{\omega}$ one can easily check that the hypotheses of Theorem 5.1 are satisfied. Hence there is a continuous mapping $F: 2^{\omega} \rightarrow \mathcal{C}$ such that $F(y) \subset X$ and $y \in \pi(F(y))$ for all $y \in Y$.

Then the set $H=\left\{(y, z) \in 2^{\omega} \times 2^{\omega}:(y, z) \in F(y)\right\}$ is compact and $\pi(H) \supset Y$; since for any $y \in Y$ we have $H \cap\left(\{y\} \times 2^{\omega}\right) \subset X$, the restriction of $\pi$ to $X^{\prime}=H \cap\left(Y \times 2^{\omega}\right) \subset X$ is a perfect mapping from $X^{\prime}$ onto $Y$.

(iii) $\Rightarrow$ (iv). This implication follows from the proof of Theorem 6.5 in [3] where given any mapping $f: X \rightarrow Y$ defined on a $\Pi_{1}^{1}$ space $X$ we construct a mapping $\tilde{f}: \widetilde{X} \rightarrow \widetilde{Y}$ with the following properties:

- $\widetilde{X}$ is a Boolean combination of $\boldsymbol{\Pi}_{2}^{0}$ sets and sets homeomorphic to $Y$.

- $\widetilde{Y}$ is the union of $Y$ and some discrete countable set.

- If $f$ is compact covering then $\tilde{f}$ is compact covering.

- If $\widetilde{f}$ is inductively perfect then $f$ is inductively perfect.

In particular if $Y$ is $\boldsymbol{\Pi}_{3}^{0}$ then it is clear that both spaces $\widetilde{X}$ and $\tilde{Y}$ are also $\boldsymbol{\Pi}_{3}^{0}$, and one can easily derive (iv) from (iii).

$(\mathrm{v}) \Rightarrow(\mathrm{i})$. Fix an arbitrary $\Pi_{1}^{1}$ subset $W$ in $2^{\omega}$. Let $h: G \rightarrow \mathcal{G}$ be the homeomorphism given by Theorem 6.2 and set $Z^{*}=h\left(W \times \omega^{\omega}\right) \subset \mathcal{K}(\mathbb{Q})$. Fix in $\mathcal{K}(\mathbb{Q})$ a relatively closed subset $Y^{*}$ which is $\check{\mathbf{P}}_{\sigma}$ but not $\mathbf{P}_{\sigma}$; this is possible by Lemma 6.1. Then the set $X^{*}=\left\{(S, T) \in Y^{*} \times Z^{*}: S \subset T\right\}$ is also $\Pi_{1}^{1}$. For simplicity we work in $2^{\omega}$ rather than in $\mathcal{K}\left(2^{\omega}\right)$. For this fix 
some homeomorphim $\psi: \mathcal{K}\left(2^{\omega}\right) \rightarrow 2^{\omega}$ and set

$$
Y=\psi\left(Y^{*}\right) \subset 2^{\omega}, \quad Z=\psi\left(Z^{*}\right) \subset 2^{\omega}, \quad X=(\psi \times \psi)\left(X^{*}\right) \subset Y \times Z,
$$

and let $\pi_{X}$ denote the canonical projection from $X$ onto $Y$.

FACT 1. If $W$ is non-Borel then $\pi_{X}$ is compact covering.

Proof. Since $W$ is non-Borel, $Z^{*}$ is a cofinal subset of $\mathcal{K}(\mathbb{Q})$ by Theorem 6.2. Let $K$ be a compact subset of $Y$. Then $\mathcal{H}=\psi^{-1}(K)$ is a compact subset of $Y^{*} \subset \mathcal{K}(\mathbb{Q})$, hence $S=\bigcup \mathcal{H}$ is a compact subset of $\mathbb{Q}$, and we can find $T \in Z^{*}$ such that $S \subset T$. Set $z=\psi(T) \in Z$; if $y \in K$ then $\psi^{-1}(y) \in \mathcal{H}$, hence $\psi^{-1}(y) \subset S \subset T$, and so $(y, z) \in X$. This shows that $K \times\{z\} \subset X$ and obviously $\pi(K \times\{z\})=K$.

Recall that a set is said to be thin if it contains no perfect nonempty subset.

FACT 2. If $W$ is thin then $\pi_{X}$ is not inductively perfect.

Proof. Suppose $\pi_{X}$ is inductively perfect and let $H$ be a closed subset of $2^{\omega} \times 2^{\omega}$ such that $\pi(X \cap H)=Y$ and $H \cap\left(\{y\} \times 2^{\omega}\right) \subset X$ for every $y \in Y$. Replacing $H$ by $\overline{H \cap X}$ if necessary, we can assume that $H \cap X$ is dense in $H$. Since $\psi^{-1}(y) \subset \psi^{-1}(z)$ for all $(y, z) \in X$, this holds for all $(y, z) \in H$.

Recall that, for two topological spaces $Y$ and $X$, a mapping $\Psi: Y \rightarrow$ $\mathcal{K}(X)$ is said to be upper semicontinuous if for any open subset $O$ of $X$, the set

$$
\Psi^{-}(O):=\{y \in Y: \Psi(y) \subset O\}
$$

is open. In particular, if $\psi$ is a function $Y \rightarrow X$, the mapping $\Psi: y \mapsto\{\psi(y)\}$ is upper semicontinuous if and only if $\psi$ is continuous.

The mapping $y \mapsto H(y)=\{z:(y, z) \in H\}$ is then upper semicontinuous from $2^{\omega}$ to $\mathcal{K}\left(2^{\omega}\right)$; furthermore, if $y \in Y$ then $H(y) \subset \psi\left(Z^{*}\right) \subset \psi(\mathcal{G})$. Since the set $\Gamma:=\psi(\mathcal{G})$ is $\mathbf{G}_{\delta}$, the set

$$
B=\{\beta \in \bar{Y}: H(\beta) \subset \Gamma\}
$$

is also $\mathbf{G}_{\delta}$ and contains $Y$.

Consider the set-valued mapping $M: \beta \mapsto h^{-1} \circ \psi^{-1}(H(\beta))$ defined on $B$. Clearly $M$ is u.s.c. with nonempty compact values in $G$; moreover $M(y) \subset W \times \omega^{\omega}$ for all $y \in Y$ since $H(y) \subset \psi\left(Z^{*}\right)$. It follows that $\{(w, \alpha, y) \in$ $\left.2^{\omega} \times \omega^{\omega} \times Y:(w, \alpha) \in M(y)\right\}$ is a closed subset of $2^{\omega} \times \omega^{\omega} \times Y$ contained in $W \times \omega^{\omega} \times Y$. Hence its projection $D$ on the first factor is an analytic subset of $W$; and since $W$ is thin, $D$ is countable. Fix some enumeration $\left(w_{k}\right)$ of $D$, and set, for all $k$,

$$
B_{k}=\left\{\beta \in B: M(\beta) \cap\left(\left\{w_{k}\right\} \times 2^{\omega}\right) \neq \emptyset\right\} .
$$

Since $M$ is u.s.c., each $B_{k}$ is closed in $B$. If $y \in Y$ then $M(y)$ is nonempty and contained in $D$; hence $Y \subset \bigcup_{k} B_{k}$. 
Conversely, if $\beta \in B \backslash Y$ then since $Y^{*}$ is a closed subset of $\mathcal{K}(\mathbb{Q})$, we have $\psi^{-1}(\beta) \in \overline{Y^{*}} \backslash Y^{*} \subset \mathcal{K}\left(2^{\omega}\right) \backslash \mathcal{K}(\mathbb{Q})$. For each $z$ such that $(\beta, z) \in H$ we have $\psi^{-1}(z) \supset \psi^{-1}(\beta)$, thus $\psi^{-1}(z) \not \subset \mathbb{Q}$, and since $W \times \omega^{\omega}=h^{-1}(\mathcal{K}(\mathbb{Q}))$, it follows that $(w, \alpha)=h^{-1} \circ \psi^{-1}(z) \notin W \times \omega^{\omega}$. But $(w, \alpha) \in 2^{\omega} \times \omega^{\omega}$, hence $w \notin W$ and $w \notin D$. We conclude that $\beta \notin \bigcup B_{k}$ if $\beta \in B \backslash Y$.

This proves that $Y=\bigcup_{k} B_{k}$ is $\mathbf{F}_{\sigma}$ in the Polish space $B$, hence $Y$ is $\mathbf{P}_{\sigma}$; and this is impossible since $Y$ is homeomorphic to $Y^{*}$ which is not $\mathbf{P}_{\sigma}$.

Thus if $W$ is any $\boldsymbol{\Pi}_{1}^{1}$ non-Borel set, then by Fact $1, \pi_{X}$ is a compact covering mapping from the $\boldsymbol{\Pi}_{1}^{1}$ space $X$ onto the $\check{\mathbf{P}}_{\sigma}$ space $Y$, hence by (v), $\pi_{X}$ is inductively perfect, and from Fact 2 we derive that $W$ is not thin. By Theorem 1.2 this proves (i), and finishes the proof of the theorem.

7.3. Theorem. The following are equivalent:

(i) $\operatorname{Det}\left(\boldsymbol{\Sigma}_{1}^{1}\right)$.

(ii) Any compact covering mapping from a $\boldsymbol{\Pi}_{1}^{1}$ space onto a $\boldsymbol{\Pi}_{1}^{1}$ space is inductively perfect.

(iii) In any $\Pi_{1}^{1}$ space $X$, any $\Pi_{1}^{1}$ cofinal subset of $\mathcal{K}(X)$ is continuously cofinal.

(iv) Any $\Pi_{1}^{1}$ cofinal subset of $\mathcal{K}(\mathbb{Q})$ admits a Borel domination function.

Proof. The implications (i) $\Rightarrow($ iii $) \Rightarrow$ (ii) follow from the remarks in Section 7 of [3].

(ii) $\Rightarrow$ (iv). If $\mathcal{A}$ is a $\Pi_{1}^{1}$ cofinal subset of $\mathcal{K}(\mathbb{Q})$ consider the set

$$
X^{*}=\{(S, T) \in \mathcal{K}(\mathbb{Q}) \times \mathcal{A}: S \subset T\} .
$$

Then as in the proof of Fact 1 in Theorem 7.2 one easily checks that the canonical projection to the first factor from $X$ onto $\mathcal{K}(\mathbb{Q})$ is compact covering, hence by assumption (ii) its restriction to some relatively closed subset $P$ of $X$ is perfect and onto. Then by classical results $P$ admits a first Baire class section, that is, a function $f: \mathcal{K}(\mathbb{Q}) \rightarrow \mathcal{A}$ of the first Baire class whose graph lies in $P$, thus satisfying $S \subset f(S)$ for all $S \in \mathcal{K}(\mathbb{Q})$, which shows that $f$ is a domination function for $\mathcal{A}$.

(iv) $\Rightarrow$ (i). Asuming (iv) we shall prove that any $\boldsymbol{\Pi}_{1}^{1}$ non-Borel set is $\boldsymbol{\Pi}_{1}^{1}$ complete by Borel reductions, which will prove (i) by Harrington's result quoted in Section 1 (see Theorem 1.3).

So fix a $\Pi_{1}^{1}$ non-Borel subset $W$ of $2^{\omega}$ and let $h$ be the homeomorphism given by Theorem 6.2. Since $W$ is not Borel, the set $\mathcal{A}=h\left(W \times \omega^{\omega}\right)$ is a $\Pi_{1}^{1}$ cofinal subset of $\mathcal{K}(\mathbb{Q})$. Hence by (iv) there exists a Borel function $f: \mathcal{K}(\mathbb{Q}) \rightarrow \mathcal{A}$ such that $S \subset f(S)$ for all $S \in \mathcal{K}(\mathbb{Q})$. Then $h^{-1} \circ f$ defines a Borel mapping from $\mathcal{K}(\mathbb{Q})$ into $W \times \omega^{\omega}$ which extends to a Borel mapping $g: B \rightarrow 2^{\omega} \times \omega^{\omega}$ defined on a Borel subset $B$ containing $\mathcal{K}(\mathbb{Q})$. 
Let $g_{0}: B \rightarrow 2^{\omega}$ denote the first coordinate function of $g$. Since $S \subset$ $f(S)=h \circ g(S)$ for all $S \in \mathcal{K}(\mathbb{Q})$, the set $B^{\prime}=\{S \in B: S \subset h \circ g(S)\}$ is Borel and contains $\mathcal{K}(\mathbb{Q})$; and for all $S \in B^{\prime}$ :

- if $S \subset \mathbb{Q}$, then $g(S) \in W \times \omega^{\omega}$ and $g_{0}(S) \in W$,

- if $S \not \subset \mathbb{Q}$, then $h \circ g(S) \supset S$ and $h \circ g(S) \notin \mathcal{K}(\mathbb{Q})$, hence $g(S) \notin W \times \omega^{\omega}$ and $g_{0}(S) \notin W$.

This proves that $\mathcal{K}(\mathbb{Q})=g_{0}^{-1}(W)$, hence $g_{0}$ is a Borel reduction of $\mathcal{K}(\mathbb{Q})$ to $W$, and since $\mathcal{K}(\mathbb{Q})$ is $\Pi_{1}^{1}$ complete, this proves that $W$ is $\Pi_{1}^{1}$-complete by Borel reductions.

We finish with some variations of the previous results. In the next two results $\pi$ denotes the canonical projection from $2^{\omega} \times 2^{\omega}$ onto the first factor; we also identify any mapping $f: A \rightarrow B$ with its graph and write $f \subset A \times B$.

7.4. Theorem. Assume that " $\forall \alpha \in \omega^{\omega}, \omega_{1}^{L(\alpha)}<\omega_{1}$ ". Let $X \subset 2^{\omega} \times 2^{\omega}$ be a $\boldsymbol{\Pi}_{1}^{1}$ set such that $Y=\pi(X)$ is $\boldsymbol{\Pi}_{3}^{0}$. If for any compact set $K \subset Y$ there exists a continuous mapping $f_{K}: K \rightarrow 2^{\omega}$ such that $f_{K} \subset X$ then there exists a continuous mapping $f: Y \rightarrow 2^{\omega}$ such that $f \subset X$.

Proof. The argument is similar to the proof of (i) $\Rightarrow$ (ii) in Theorem 7.2 and we sketch it briefly: apply Theorem 5.1 with $R$ as in the proof of (i) $\Rightarrow$ (ii) in Theorem 7.2 above and

$$
Z=\left\{H \in \mathcal{K}\left(2^{\omega} \times 2^{\omega}\right): H \subset X \text { and } H \text { is a graph }\right\} .
$$

Notice that the condition " $H$ is a graph" is Borel (in fact $\Pi_{2}^{0}$ ), hence $Z$ is $\boldsymbol{\Pi}_{1}^{1}$; and one easily checks that hypothesis (b) of Theorem 5.1 holds. Then the conclusion of this theorem provides a continuous mapping $F: Y \rightarrow Z$ with graph contained in $R$. Then the set $H_{0}:=\{(y, z):(y, z) \in F(y)\}$ is the graph of some function $\tilde{f}: 2^{\omega} \rightarrow 2^{\omega}$, which is continuous since $H_{0}$ is compact. Then $f=\left.\widetilde{f}\right|_{Y}: Y \rightarrow 2^{\omega}$ is continuous and $f \subset X$.

By similar arguments one can prove:

7.5. Theorem. Assume "Det $\left(\boldsymbol{\Sigma}_{1}^{1}\right)$ ". Let $X \subset 2^{\omega} \times 2^{\omega}$ be a $\boldsymbol{\Pi}_{1}^{1}$ set such that $Y=\pi(X)$ is $\boldsymbol{\Pi}_{1}^{1}$. If for any compact set $K \subset Y$ there exists a continuous mapping $f_{K}: K \rightarrow 2^{\omega}$ such that $f_{K} \subset X$ then there exists a continuous mapping $f: Y \rightarrow 2^{\omega}$ such that $f \subset X$.

7.6. Extensions and remarks. We now give some variations and extensions of the previous results. The proofs that we omit use essentially either the same arguments or some complementary ideas from [2] and [3].

1) All the results clearly have effective versions. Notice however that in Theorem 5.1 the function $f$ of the conclusion is only continuous and not 
recursive. Similarly the continuous or Borel domination functions considered in Theorem 7.3 are not effective.

2) Examining the arguments of Theorem 7.2 one easily sees that the converse of Theorem 7.4 holds, that is, one can derive the assumption " $\forall \alpha \in \omega^{\omega}, \omega_{1}^{L(\alpha)}<\omega_{1}$ " from the rest of the statement. This is also true for Theorem 7.5.

3) One can add to the list of equivalent propositions in Theorem 7.2 the following analog of statement (iv) of Theorem 7.1 and of statement (iii) of Theorem 7.3:

- In any $\Pi_{1}^{1}$ space $X$ (or in $X=\mathbb{Q}$ ), for any $\Pi_{1}^{1}$ cofinal subset $\mathcal{A}$ of $\mathcal{K}(X)$ and any $\Pi_{3}^{0}$ subset $\mathcal{B}$ of $\mathcal{K}(X)$ there exists a continuous mapping $f: \mathcal{B} \rightarrow \mathcal{A}$ satisfying $f(S) \supset S$ for all $S \in \mathcal{B}$.

But one cannot, as in the other theorems, replace here "continuous" by "Borel". Also one cannot replace the cofinality hypothesis on $\mathcal{A}$ by the more natural but weaker hypothesis " $\forall S \in \mathcal{B}, \exists T \in \mathcal{A}, S \subset T$ ".

4) For simplicity we focused on $\Pi_{1}^{1}$ cofinal subsets of $\mathcal{K}(X)$. In fact all the previous results are still valid for $\boldsymbol{\Sigma}_{2}^{1}$ cofinal subsets. This can be seen either by slight modifications in the arguments, or by using the homeomorphic embedding $\Phi: \mathcal{K}(X) \times 2^{\omega} \rightarrow \mathcal{K}(X)$ constructed in Lemma 6.6, which enables one to reduce the study of $\Sigma_{2}^{1}$ cofinal subsets to the study of $\Pi_{1}^{1}$ cofinal subsets (see the proof of Theorem 5.2 in [3]).

But in Theorems 7.4 and 7.5, one cannot replace the hypothesis " $X \Pi_{1}^{1}$ " by " $X \Sigma_{2}^{1}$ ".

5) Most of the classes considered in this work are Wadge classes. We recall that $\mathcal{Y}$ is a Wadge class if there exists $Y_{0}$ in $\mathcal{Y}$ such that any space $Y$ in $\mathcal{Y}$ is of the form $Y=\varphi^{-1}\left(Y_{0}\right)$ for some continuous mapping $\varphi: 2^{\omega} \rightarrow 2^{\omega}$; but then the mapping $\psi: Y \rightarrow Y_{0}$, obtained by restricting the domain and the range of $\varphi$, is clearly perfect. For example one can take

$$
Y_{0}=\omega^{\omega}, \mathbb{Q} \times \omega^{\omega},\left(2^{\omega} \times 2^{\omega}\right) \backslash\left(\mathbb{Q} \times \omega^{\omega}\right), \mathbb{Q}^{\omega}, \mathcal{K}(\mathbb{Q})
$$

if

$$
\mathcal{Y}=\mathbf{\Pi}_{2}^{0}, \mathbf{P}_{\sigma}, \check{\mathbf{P}}_{\sigma}, \boldsymbol{\Pi}_{3}^{0}, \boldsymbol{\Pi}_{1}^{1} .
$$

If we now consider any mapping $f: X \rightarrow Y$ it is straightforward to check the following properties:

- If $f$ is compact covering then $\psi \circ f$ is compact covering.

- If $\psi \circ f$ is inductively perfect then $f$ is inductively perfect.

It then follows that for a given space $X$ :

- If any compact covering mapping from $X$ onto $Y_{0}$ is inductively perfect then the same holds for any mapping from $X$ onto any space in $\mathcal{Y}$. 
Applying this observation to the particular classes considered in Theorems 7.1-7.3, one obtains new equivalent statements in these theorems.

6) It is interesting to compare the following statements:

(a) Any compact covering mapping $f: X \rightarrow Y$ with $X$ Borel and $Y \boldsymbol{\Sigma}_{2}^{0}$ is inductively perfect.

(A) Any compact covering mapping $f: X \rightarrow Y$ with $X$ Borel and $Y \mathbf{\Pi}_{2}^{0}$ is inductively perfect.

(b) Any compact covering mapping $f: X \rightarrow Y$ with $X \Pi_{2}^{0}$ is inductively perfect.

(B) Any compact covering mapping $f: X \rightarrow Y$ with $X \boldsymbol{\Pi}_{3}^{0}$ is inductively perfect.

As mentioned in the introduction, (a) and (b) are theorems in ZFC; whereas by Theorem 7.1, (A) is equivalent to " $\forall \alpha \in \omega^{\omega}$, $\omega^{\omega} \cap L$ is $\leq^{*}$ bounded"; and by Theorem 7.2, (B) is equivalent to " $\forall \alpha \in \omega^{\omega}, \omega^{\omega} \cap L$ is countable".

7) The results of this paper give strong evidence for the following

Conjecture. Assume that " $\forall \alpha \in \omega^{\omega}, \omega_{1}^{L(\alpha)}<\omega_{1}$ ". Then any compact covering mapping $f: X \rightarrow Y$ between two Borel spaces $X$ and $Y$ is inductively perfect.

Proving this would solve Problem 1 completely, since by statements (ii) and (iii) of Theorem 7.2 the assumption " $\forall \alpha \in \omega^{\omega}, \omega_{1}^{L(\alpha)}<\omega_{1}$ " is necessary even if one restricts the conjecture to mappings between $\Pi_{3}^{0}$ spaces.

Notice that, as in the proof of Theorem 7.2, one obtains an equivalent form of this conjecture if the hypothesis " $X$ and $Y$ are Borel" is replaced by one of the following alternative hypotheses:

- " $X$ is $\Pi_{1}^{1}$ and $Y$ is Borel",

- " $X$ is Borel" (with no assumption on $Y$ ).

Other equivalent forms can be obtained in terms of $\boldsymbol{\Pi}_{1}^{1}$ cofinal subsets of $\mathcal{K}(X)$ as in Remark 3 above, where $\mathcal{B}$ would now be any Borel (or even analytic) subset of $\mathcal{K}(X)$.

8) Finally we recall that inductively perfect mappings preserve Borel (additive or multiplicative) classes (see [2]); and as mentioned in the proof of Theorem 7.2 this property extends to compact covering mappings if one assumes that " $\forall \alpha \in \omega^{\omega}, \omega_{1}^{L(\alpha)}<\omega_{1}$ ".

But the following problem, raised by Ostrovskiu [8], is still open in ZFC:

Problem. Is the image of a Borel space under a compact covering mapping also Borel? 


\section{References}

[1] J. P. R. Christensen, Necessary and sufficient conditions for the measurability of certain sets of closed sets, Math. Ann. 200 (1973), 189-193.

[2] G. Debs and J. Saint Raymond, Compact covering and game determinacy, Topology Appl. 68 (1996), 153-185.

[3] - - - Cofinal $\boldsymbol{\Sigma}_{1}^{1}$ and $\boldsymbol{\Pi}_{1}^{1}$ subsets of $\omega^{\omega}$, Fund. Math. 159 (1999), 161-193.

[4] L. Harrington, Analytic Determinacy and $0^{\sharp}$, J. Symbolic Logic 43 (1978), 685-693.

[5] W. Just and H. Wicke, Some conditions under which tri-quotient or compactcovering maps are inductively perfect, Topology Appl. 55 (1994), 289-305.

[6] A. S. Kechris, A. Louveau and W. H. Woodin, The structure of $\sigma$-ideals of compact sets, Trans. Amer. Math. Soc. 301 (1987) 263-288.

[7] Y. N. Moschovakis, Descriptive Set Theory, North-Holland, Amsterdam, 1980.

[8] A. V. Ostrovskiǔ, New classes of maps connected with compact covering maps, Vestnik Moskov. Gos. Univ. 1994, no. 4, 24-28 (in Russian).

[9] J. Saint Raymond, Caractérisation d'espaces polonais, Séminaire d'Initiation à l'Analyse, 11-12èmes années, no. 5 (1971-73).

[10] —, La structure borélienne d'Effros est-elle standard?, Fund. Math. 100 (1978), 201-210.

[11] R. L. Sami, Analytic Determinacy and $0^{\sharp}$. A forcing-free proof of Harrington's theorem, Fund. Math. 160 (1999), 153-159.

[12] W. Sierpiński, Sur les images biunivoques de l'ensemble de tous les nombres rationnels, Mathematika 1 (1929), 18-21.

[13] R. M. Solovay, On the cardinality of $\Sigma_{2}^{1}$ sets of reals, in: Foundations of Mathematics (Symposium commemorating Kurt Gödel, Columbus, OH, 1966), Springer, New York, 1969, 58-73.

Equipe d'Analyse

Université Paris 6

Boîte 186

4, place Jussieu

75252 Paris CEDEX 05, France

E-mail: gad@ccr.jussieu.fr jsr@ccr.jussieu.fr 